\title{
Article \\ AI Models for Predicting Readmission of Pneumonia Patients within 30 Days after Discharge
}

\author{
Jiin-Chyr Hsu ${ }^{1,2,+}$, Fu-Hsing Wu ${ }^{3,+}$, Hsuan-Hung Lin ${ }^{4}\left(\mathbb{D}\right.$, Dah-Jye Lee ${ }^{5}(\mathbb{D})$, Yung-Fu Chen ${ }^{3,4,6, *(D)}$ \\ and Chih-Sheng Lin ${ }^{1, *}$
}

check for updates

Citation: Hsu, J.-C.; Wu, F.-H.; Lin, H.-H.; Lee, D.-J.; Chen, Y.-F.; Lin, C.-S. AI Models for Predicting Readmission of Pneumonia Patients within 30 Days after Discharge. Electronics 2022, 11, 673. https:// doi.org/10.3390/electronics11050673

Academic Editor: Gwanggil Jeon

Received: 30 December 2021

Accepted: 20 February 2022

Published: 22 February 2022

Publisher's Note: MDPI stays neutral with regard to jurisdictional claims in published maps and institutional affiliations.

Copyright: (c) 2022 by the authors. Licensee MDPI, Basel, Switzerland. This article is an open access article distributed under the terms and conditions of the Creative Commons Attribution (CC BY) license (https:// creativecommons.org/licenses/by/ $4.0 /)$.
1 Department of Radiology, BenQ Medical Center, The Affiliated BenQ Hospital of Nanjing Medical University, Nanjing 210017, China; jinchyr.hsu@msa.hinet.net

2 Department of Internal Medicine, Taipei Hospital, Ministry of Health and Welfare, New Taipei 24213, Taiwan

3 Department of Health Services Administration, China Medical University, Taichung 406040, Taiwan; raxwu@mail.cmu.edu.tw

4 Department of Management Information Systems, Central Taiwan University of Science and Technology, Taichung 406053, Taiwan; shlin@ctust.edu.tw

5 Department of Electrical and Computer Engineering, Brigham Young University, Provo, UT 84602, USA; djlee@byu.edu

6 Department of Dental Technology and Materials Science, Central Taiwan University of Science and Technology, Taichung 406053, Taiwan

* Correspondence: yfchen@ctust.edu.tw (Y.-F.C.); chihsheng.lin@benqmedicalcenter.com (C.-S.L.)

+ These authors contributed equally to this study.

\begin{abstract}
A model with capability for precisely predicting readmission is a target being pursued worldwide. The objective of this study is to design predictive models using artificial intelligence methods and data retrieved from the National Health Insurance Research Database of Taiwan for identifying high-risk pneumonia patients with 30-day all-cause readmissions. An integrated genetic algorithm (GA) and support vector machine (SVM), namely IGS, were used to design predictive models optimized with three objective functions. In IGS, GA was used for selecting salient features and optimal SVM parameters, while SVM was used for constructing the models. For comparison, logistic regression (LR) and deep neural network (DNN) were also applied for model construction. The IGS model with AUC used as the objective function achieved an accuracy, sensitivity, specificity, and area under ROC curve (AUC) of 70.11\%, 73.46\%, 69.26\%, and 0.7758, respectively, outperforming the models designed with LR $(65.77 \%, 78.44 \%, 62.54 \%$, and 0.7689 , respectively) and DNN $(61.50 \%$, $79.34 \%, 56.95 \%$, and 0.7547 , respectively), as well as previously reported models constructed using thedata of electronic health records with an AUC of 0.71-0.74. It can be used for automatically detecting pneumonia patients with a risk of all-cause readmissions within 30 days after discharge so as to administer suitable interventions to reduce readmission and healthcare costs.
\end{abstract}

Keywords: pneumonia readmission; imbalanced dataset; integrated genetic algorithm and support vector machine (IGS); logistic regression (LR); deep neural network (DNN)

\section{Introduction}

Readmission refers to patients who have been admitted to inpatient wards again after being discharged from hospitals within a short period of time. It may be attributed to unsuccessful treatments, new diseases, worsening comorbidities, or degraded quality of care [1], and can be caused by clinical and non-clinical factors [2,3], resulting in increased healthcare cost. The non-clinical factors include poor social support, housing instability, and drug abuse [2], whereas the clinical factors are related to patents having a high Charlson comboridity index, using 10 or more medications, and living in a community with home care [3]. The readmission rate is generally considered as an indicator for evaluating the healthcare quality of a hospital [4], although it has been challenged that substantial errors were found when using it as a marker of healthcare quality [5]. In addition to improving 
hospital quality, the hospital readmission reduction program (HRRP) has also been shown to be useful for reducing healthcare cost and elevating patient satisfaction [6].

\subsection{Policies for Reducing Readmissions}

Appropriate policies, including financial incentives [7-9], care transition processes [10], and health information exchange (HIE) [11], as well as improved nursing environments [12] and integrated skilled nursing facilities (SNF) [8], are effective at decreasing patient readmissions. For example, due to the recent passage of US legislation of imposing financial penalties on hospitals with excessive patient readmissions, a system was designed to identify chronic obstructive pulmonary disease (COPD) patients who had a higher probability and higher healthcare cost of readmission for post-discharge interventions so as to save aftercare cost [9]. Compared to patients provided with costly aftercare, it saved $90 \%$ healthcare cost by identifying COPD patients with a high potential readmission and potentially the highest care cost from discharge summaries in order to perform readmission mitigation interventions [9]. Financial incentives provided by Medicare's HRRP in the U.S. were also reported to have reduced the readmission rate by $0.3-1.2 \%$ for each of the five HRRPtargeted conditions, i.e., acute myocardial infarction, heart failure, COPD, pneumonia, and hip and/or knee surgery [7].

Care transition processes were also shown to be useful to reduce the risk standardized readmission rate (RSRR); among the 20 care transition processes evaluated in a study performed at ten veterans affairs hospitals in the U.S., more care transition processes performed before and after patient discharge could achieve a lower RSRR [10]. Additionally, health information exchange (HIE) is also a good strategy for reducing the readmission rate; hospitals are suggested to adopt HIE to exchange health information with primary care providers for the collaboration of patient care in order to reduce readmission rate [11].

In [12], the relationship between patient readmission and hospital nursing factors, including work environment, staffing level, and education, were studied and it was found that nurse work environment and staffing level were related to 30-day readmission among $23.2 \%$ heart failure, $19.1 \%$ acute myocardial infarction, and $17.8 \%$ pneumonia patients. Excessive nurse's workload with one more patient per nurse was found to be related to a $7 \%, 9 \%$, and $6 \%$ higher chance of readmission for heart failure, acute myocardial infarction, and pneumonia patients, respectively; furthermore, a good hospital healthcare environment was also related to a $7 \%, 6 \%$, and $10 \%$ lower chance of readmission for heart failure, acute myocardial infarction, and pneumonia patients, respectively, when compared to a poor environment [12]. By analyzing the data of hospital and year fixed effects for both pneumonia and heart failure, except for investor-owned hospital, the vertical SNF integration was shown to be significantly associated with a reduction of 30-day pneumonia readmission in other types of hospitals. However, vertical SNF integration was not significantly associated in all types of hospitals for the reduction of 30-day heart failure readmission [8].

\subsection{Factors Associated with Readmissions and Interventions for High-Risk Patients}

Demographic (gender, age, income, resident region, and education), treatment and clinical (principal diagnosis, treatment department, surgery, clinical test results, number of accompanied treatments, number of comorbidities, depression, and mental health status), and healthcare utilization (length of hospital stay, number of out-patient visits, frequency of hospital admission, type of insurance, type of patient room, frequency of emergency visit, and route of admission) factors were reported to be the greatest risks for readmission [13-15]. As observed in [14], factors including unemployment, less than a high school education, and diagnosed diseases (chronic obstructive pulmonary disease and coronary artery disease) were found to be independent factors associated with readmissions. In [15], other diseases, including infection, neoplasm, heart failure, gastrointestinal disorder, and liver disorder, were also reported to be the most frequent primary diagnoses of potentially avoidable readmissions. Patient's social risk factors and the community's social determi- 
nant of health were also reported to effect the readmission rate in [16]. It was suggested that the readmission performance of the CMS HRRP readmission model may be improved by including factors of patient social risk and community social determinant of health, thereby removing unjustified penalties on hospitals located at geographic areas accommodating a large proportion of high-risk patients [16]. Hence, after being discharged, the comorbidities of high-risk patients should be carefully cared for by providing appropriate interventions in high-risk patients for effectively reducing readmission rate. It was noted that even simple interventions, such as telephonic case management, were useful for reducing all-cause readmissions in high-risk patients prioritized based on episodic risk group (ERG) score after discharge [17].

Charles et al. [18] adopted the LACE index to screen high-risk older patients in an acute care hospital for care coordination intervention by providing medications, equipment, and homecare services to the high-risk group, achieving lower rates of 30-day ED revisits $(30.5 \%)$, as well as 90 -day $(39.3 \%)$ and 6-month (50.9\%) readmissions, when compared to the non-intervention group (33.3\%, 44.6\%, and 58.4\%, respectively). Although detecting patients with a high-risk readmission accompanied with a high care cost for aftercare intervention is useful to save hospital healthcare cost, patient safety is generally deemed as the main concern of HRRP. A clinical decision support system (CDSS) provides useful information and expert knowledge to improve the diagnostic performance, treatment outcome, or healthcare quality in the clinical setting [19], and has been widely applied in the detection of medical events [20-32]. In this study, we aimed to design a CDSS model for predicting readmissions of high-risk patients admitted with pneumonia, so that post-discharge intervention can be administrated to prevent all-cause readmissions and elevate patient safety.

\subsection{AI Models for Predicting Associated Events of Hospital Admission and Readmission}

Recent studies that have focused on constructing AI models for predicting associated events of hospital admission are summarized in Table 1. In [33], a model integrating multilayer perceptron (MLP) and convolutional neural network (CNN), input with 24 numeric and categorical features, as well as four text notes at a triage of emergency departments (ED), respectively, was proposed for predicting hospital admission, with a performance reaching an AUC of 0.83. In [34], by analyzing the effectiveness of various machine learning (ML) models for predicting mortality, critical care outcome, and the need for hospitalization of ED patients reported in 11 studies, and showed that deep neural network (DNN) [35-37] and extreme gradient boosting (XGBoost) [37-39] achieved the greatest predictive accuracy among the assessed ML models, with an AUC of 0.782-0.92 and 0.922-0.962, respectively.

Table 1. Recent AI models for predicting associated events of hospital admissions.

\begin{tabular}{|c|c|c|c|c|c|c|}
\hline $\begin{array}{l}\text { Study } \\
\text { (Year) }\end{array}$ & $\begin{array}{l}\text { Prediction } \\
\text { Event }\end{array}$ & Method & Dataset & $\begin{array}{l}\text { Used Features or } \\
\text { Input }\end{array}$ & $\begin{array}{c}\text { Predictive } \\
\text { Performance }\end{array}$ & Issue \\
\hline $\begin{array}{c}{[33]} \\
(2020)\end{array}$ & $\begin{array}{c}\text { Hospital } \\
\text { admission at } \\
\text { ED }\end{array}$ & $\begin{array}{c}\text { MLP with } \\
\text { numeric and } \\
\text { categorical } \\
\text { features + CNN } \\
\text { with textual } \\
\text { data }\end{array}$ & $\begin{array}{l}\text { 260,000 ED records } \\
\text { of a hospital in } \\
\text { France collected } \\
\text { within 2015-2019 }\end{array}$ & $\begin{array}{l}28 \text { features of } \\
\text { numeric, categorical, } \\
\text { and textual data }\end{array}$ & $\mathrm{AUC}=0.83$ & \\
\hline $\begin{array}{l}{[40]} \\
(2020)\end{array}$ & $\begin{array}{l}\text { Daily hospital } \\
\text { admission due } \\
\text { to respiratory- } \\
\text { and circulatory- } \\
\text { related } \\
\text { disorders }\end{array}$ & $\mathrm{LSTM}+\mathrm{CNN}$ & $\begin{array}{l}\text { Patients } \geq 65 \text { y due } \\
\text { to circulatory or } \\
\text { respiratory } \\
\text { disorders across the } \\
\text { region of Madrid, } \\
\text { Spain, within } \\
2001-2013\end{array}$ & $\begin{array}{l}13 \text { locations and } \\
12 \text { features of } \\
\text { chemical air } \\
\text { pollutants, weather } \\
\text { observations, and } \\
\text { pollen observations }\end{array}$ & $\begin{array}{l}\text { RMSE }=11.21 \\
\text { and } 11.76 \text { for } \\
\text { circulatory and } \\
\text { respiratory } \\
\text { cases, } \\
\text { respectively }\end{array}$ & $\begin{array}{c}\text { Patients } \\
\text { with } \\
\text { age }<65 \text { y } \\
\text { were } \\
\text { excluded }\end{array}$ \\
\hline
\end{tabular}


Table 1. Cont.

\begin{tabular}{|c|c|c|c|c|c|c|}
\hline $\begin{array}{l}\text { Study } \\
\text { (Year) }\end{array}$ & $\begin{array}{l}\text { Prediction } \\
\text { Event }\end{array}$ & Method & Dataset & $\begin{array}{l}\text { Used Features or } \\
\text { Input }\end{array}$ & $\begin{array}{l}\text { Predictive } \\
\text { Performance }\end{array}$ & Issue \\
\hline $\begin{array}{c}{[41]} \\
(2021)\end{array}$ & $\begin{array}{c}\text { ICU admission } \\
\text { for COVID-19 } \\
\text { patients }\end{array}$ & $\begin{array}{l}\text { COVID-Net } \\
\text { Clinical ICU }\end{array}$ & $\begin{array}{l}1925 \text { COVID-19 } \\
\text { patient records } \\
\text { retrieved from a } \\
\text { hospital in Canada } \\
\text { in } 2020\end{array}$ & $\begin{array}{l}228 \text { clinical features } \\
\text { in fields of } \\
\text { demographic } \\
\text { information, } \\
\text { previous diseases, } \\
\text { blood results, and } \\
\text { vital signs for each } \\
\text { patient }\end{array}$ & $\mathrm{ACC}=96.9 \%$ & \\
\hline [42] (2021) & $\begin{array}{l}\text { In-hospital cost } \\
\text { and LOS of } \\
\text { admitted } \\
\text { patients }\end{array}$ & RUP & $\begin{array}{c}\text { 750,000 EMRs of } \\
\text { discharged patients } \\
\text { from } 2012 \text { to } 2015 \\
\text { collected from a } \\
\text { hospital quality } \\
\text { monitoring system } \\
\text { of China }\end{array}$ & $\begin{array}{l}\text { Patient features, } \\
\text { diagnosis texts, } \\
\text { operation texts, } \\
\text { diagnosis IDs, and } \\
\text { operation IDs }\end{array}$ & $\begin{array}{l}\text { RMSE }=7765 \\
\text { CNY and } \\
7.056 \text { days for } \\
\text { cost and LOS } \\
\text { predictions } \\
\text { respectively }\end{array}$ & \\
\hline $\begin{array}{c}{[43]} \\
(2020)\end{array}$ & $\begin{array}{c}\text { LOS for } \\
\text { cardiovascular } \\
\text { hospitalization } \\
\text { in ICU }\end{array}$ & $\begin{array}{l}\text { Stacking } \\
\text { regression }\end{array}$ & $\begin{array}{l}\text { Health data of } \\
\text { 61,532 ICU stays in } \\
\text { the MIMIC-III } \\
\text { dataset provided } \\
\text { by MIT Lab }\end{array}$ & $\begin{array}{l}\text { Demographics, vital } \\
\text { signs, laboratory } \\
\text { tests, medications, } \\
\text { and more clinical } \\
\text { variables }\end{array}$ & $\begin{array}{l}\text { MAE }= \\
1.92 \text { days }\end{array}$ & \\
\hline
\end{tabular}

In [40], independent meteorological, pollen, and chemical pollution data were adopted to design predictive models using long short-term memories and CNN (LSTM + CNN) to forecast daily hospital admissions for patients due to respiratory- and circulatory-related disorders, which showed that the models could precisely forecast hospital admissions with a root mean squared error (RMSE) of 11.21 and 11.76 for circulatory and respiratory cases, respectively. Moreover, in [41], a neural network, namely COVID-Net Clinical ICU, was proposed to predict admission to intensive care units (ICU) for COVID patients with an accuracy of $96.9 \%$.

In [42], a deep in-hospital resource utilization prediction (RUP) approach with multitask learning from electronic medical records (EMRs) was proposed to estimate the inhospital cost and length of stay (LOS) of admitted patients. Inputs for the multi-task learning included patient features, diagnosis/operation texts, and the diagnosis/operation IDs. The performance of the RUP model reached an RMSE of 7765 CNY and 7.056 days for in-hospital cost and LOS, respectively. Additionally, in [43], stacking regression was shown to outperform DNN, gradient boosting regression (GBR), and random forest in predicting LOS for cardiovascular hospitalization in the ICU, with a mean average error (MAE) of 1.92 days.

Recent studies on predicting associated events of hospital readmissions are summarized in Table 2. In [44], a trajectory-based deep learning (TADEL) method was proposed to capture series of admissions in the medical history for representing the patient's readmission trajectory for being input to train the deep-learning model, reaching a predictive performance with a recall, precision, F1 score, and AUC of 99.3\%, 77.9\%, 87.3\%, and 0.884, respectively, for predicting all-cause readmission. 
Table 2. Recent AI models for predicting hospital readmissions.

\begin{tabular}{|c|c|c|c|c|c|c|}
\hline $\begin{array}{l}\text { Study } \\
\text { (Year) }\end{array}$ & $\begin{array}{l}\text { Prediction } \\
\text { Event }\end{array}$ & Method & Dataset & $\begin{array}{l}\text { Categories of } \\
\text { Features }\end{array}$ & AUC & Issue \\
\hline $\begin{array}{c}{[44]} \\
(2021)\end{array}$ & $\begin{array}{l}\text { 30-day } \\
\text { hospital } \\
\text { readmission }\end{array}$ & $\begin{array}{l}\text { TADEL by } \\
\text { capturing } \\
\text { dynamic } \\
\text { medical history }\end{array}$ & $\begin{array}{c}\text { A balanced dataset } \\
\text { of } 72,668 \\
\text { readmission and } \\
72,663 \\
\text { non-readmission } \\
\text { patients acquired } \\
\text { from national } \\
\text { Medicare claims of } \\
\text { all hospitals in the } \\
\text { US from } 2011 \text { to } \\
2015\end{array}$ & $\begin{array}{l}\text { Health status } \\
\text { factors, insurance } \\
\text { coverage and } \\
\text { payment, history of } \\
\text { health service } \\
\text { utilizations and } \\
\text { hospitalizations, } \\
\text { and } \\
\text { sociodemographic } \\
\text { information }\end{array}$ & 0.884 & $\begin{array}{l}\text { Using a balanced } \\
\text { dataset for testing } \\
\text { is not the real } \\
\text { situation in } \\
\text { practice, the dataset } \\
\text { is usually very } \\
\text { imbalanced, which } \\
\text { may degrade the } \\
\text { predictive } \\
\text { performance }\end{array}$ \\
\hline $\begin{array}{c}{[45]} \\
(2018)\end{array}$ & $\begin{array}{l}\text { 90-day } \\
\text { hospital } \\
\text { readmission }\end{array}$ & $\mathrm{GBM}+\mathrm{GA}$ & $\begin{array}{l}69,984 \text { encounters } \\
\text { retrieved from } \\
\text { 10-year dataset of } \\
\text { 130 US hospitals }\end{array}$ & $\begin{array}{c}55 \text { attributes } \\
\text { (including HbA1c, } \\
\text { gender, discharge } \\
\text { disposition, } \\
\text { admission source, } \\
\text { specialty of the } \\
\text { admitting } \\
\text { physician, primary } \\
\text { diagnosis (9), race, } \\
\text { age, time in } \\
\text { hospital, etc.) }\end{array}$ & $\begin{array}{c}\text { Not shown, } \\
\text { ACC = } \\
97.05 \%\end{array}$ & AUC not shown \\
\hline $\begin{array}{l}{[46]} \\
(2018)\end{array}$ & $\begin{array}{c}\text { 30-day } \\
\text { hospital } \\
\text { readmission, } \\
\text { etc. }\end{array}$ & $\begin{array}{c}\text { SVM + feature } \\
\text { selection } \\
\text { algorithm } \\
\text { (EMOBPSO), } \\
\text { etc. }\end{array}$ & $\begin{array}{l}2871 \text { and } 40,460 \\
\text { readmission and } \\
\text { non-readmission } \\
\text { cases from the HIS } \\
\text { of a hospital in } \\
\text { northeast China }\end{array}$ & $\begin{array}{c}21 \text { fields of } 3 \\
\text { databases } \\
\text { (outpatient } \\
\text { information, EMR, } \\
\text { and inpatient } \\
\text { information) in the } \\
\text { HIS }\end{array}$ & 0.9038 & $\begin{array}{l}\text { Low precision } \\
\qquad(43.43 \%)\end{array}$ \\
\hline $\begin{array}{c}{[47]} \\
(2020)\end{array}$ & $\begin{array}{l}\text { hospital } \\
\text { readmissions }\end{array}$ & $\begin{array}{c}\text { JICFS } \\
\text { (including } \\
\ell_{1} \text {-norm } \\
\text { regularization } \\
\text { for } \\
\text { class-imbalance } \\
\text { aware feature } \\
\text { selection) }\end{array}$ & $\begin{array}{l}6 \text { open readmission } \\
\text { datasets (all-cause, } \\
\text { LACE-score, } \\
\text { MIMIC, T-carer, } \\
\text { RA, and diabetic) }\end{array}$ & 15-243 features & $\begin{array}{l}0.733- \\
0.9299\end{array}$ & $\begin{array}{c}\text { Low MCC for } \\
2 \text { datasets ranging } \\
\text { from } 0.5012-0.546\end{array}$ \\
\hline $\begin{array}{c}{[48]} \\
(2021)\end{array}$ & $\begin{array}{l}\text { hospital } \\
\text { readmissions }\end{array}$ & Graph-CL & $\begin{array}{c}6 \text { open readmission } \\
\text { datasets (All-cause, } \\
\text { LACE-score, } \\
\text { MIMIC, T-carer, } \\
\text { RA, and diabetic) }\end{array}$ & $\begin{array}{l}\text { Adopted 15-75 } \\
\text { features }\end{array}$ & $0.776-0.886$ & $\begin{array}{c}\text { Low MCC for } \\
3 \text { datasets ranging } \\
\text { from } 0.561-0.617\end{array}$ \\
\hline $\begin{array}{c}{[49]} \\
(2019)\end{array}$ & $\begin{array}{l}\text { 30-day ICU } \\
\text { readmission }\end{array}$ & MLP & $\begin{array}{l}\text { MIMIC-III dataset } \\
\text { with } 42,307 \text { ICU } \\
\text { stays of } 31,749 \\
\text { patients } \\
\text { from a US hospital } \\
\text { in } 2001 \text { to } 2012\end{array}$ & 12 features & 0.642 & \\
\hline
\end{tabular}


Table 2. Cont.

\begin{tabular}{|c|c|c|c|c|c|c|}
\hline $\begin{array}{l}\text { Study } \\
\text { (Year) }\end{array}$ & $\begin{array}{l}\text { Prediction } \\
\text { Event }\end{array}$ & Method & Dataset & $\begin{array}{l}\text { Categories of } \\
\text { Features }\end{array}$ & AUC & Issue \\
\hline $\begin{array}{c}{[50]} \\
(2021)\end{array}$ & $\begin{array}{l}\text { 30-day } \\
\text { hospital } \\
\text { readmission }\end{array}$ & $\begin{array}{c}\text { GBM (AI } \\
\text { model) + CDM } \\
\text { (for applying } \\
\text { trained AI } \\
\text { model to } \\
\text { multiple } \\
\text { institutions) }\end{array}$ & $\begin{array}{c}106,304 \\
\text { hospitalizations } \\
\text { with 32,242 } \\
\text { readmissions } \\
\text { retrieved from EHR } \\
\text { of Seoul National } \\
\text { University Hospital } \\
\text { in 2017-2018, etc. }\end{array}$ & $\begin{array}{l}\text { Demographics, } \\
\text { clinical index score, } \\
\text { diagnosis, } \\
\text { medication, visit } \\
\text { records, surgeries, } \\
\text { and clinical } \\
\text { examination test. }\end{array}$ & 0.8414 & $\begin{array}{l}\text { (1) Precise features } \\
\text { adopted for model } \\
\text { creation and } \\
\text { prediction are not } \\
\text { clear; } \\
\text { (2) predictive } \\
\text { parameters except } \\
\text { AUC are not } \\
\text { shown; } \\
\text { (3) the predictive } \\
\text { performance } \\
\text { degrades when } \\
\text { applying the model } \\
\text { trained in a } \\
\text { hospital to another } \\
\text { hospital }\end{array}$ \\
\hline
\end{tabular}

$\mathrm{MCC}=(\mathrm{TP} \times \mathrm{TN}-\mathrm{FP} \times \mathrm{FN}) / \sqrt{(\mathrm{TP}+\mathrm{FP})(\mathrm{TP}+\mathrm{FN})(\mathrm{TN}+\mathrm{FP})(\mathrm{TN}+\mathrm{FN})}$

In [45], an integrated gradient boosting machine with a genetic algorithm (GBM + GA) was applied to design a model for predicting 90-day hospital readmission with an accuracy as high as $97.05 \%$. In [46], an enhanced version of the multi-objective bare-bones particle swarm optimization (EMOBPSO) method, which integrated machine learning models with feature selection algorithms, was proposed for constructing the model, and it reached a predictive performance of AUC $=0.9038$ and precision $=43.43 \%$. In [47], a joint imbalanced classification and feature selection (JICFS) algorithm, which included $\ell_{1}$-norm regularization for class-imbalance aware feature selection, was proposed for constructing models for predicting readmissions using six open datasets, and it reached a predictive performance of AUC $=0.733-0.9299$.

The graph-based method, which creates a similar AI model concept used for graph or image recognition, was also proposed for designing models for readmission prediction. For example, in [48], the graph-based class-imbalance learning (graph-CL) method was adopted for constructing within-class graphs (for positive and negative samples) as well as a between-class graph for learning the pattern discrimination from within-class and between-class samples, and it reached a predictive performance of AUC $=0.776-0.886$ for predicting readmission.

\subsection{State-of-the-Art Models for the Prediction of Pneumonia Readmissions}

In [51], the predictive performances of 11 models in seven studies for predicting pneumonia readmission were reviewed. It was observed that the average rate of pneumonia readmission reported in these studies was $17.3 \%$, showing a high readmission rate for patients admitted with pneumonia after being discharged. The predictive performances of the aforementioned models exhibited an AUC ranging from 0.59 to 0.77 , with an average of 0.63 [51]. Table 3 compares the state-of-the-art studies on the prediction of readmissions for pneumonia patients. 
Table 3. Comparison of state-of-the-art studies in the prediction of readmissions for pneumonia patients.

\begin{tabular}{|c|c|c|c|c|c|c|}
\hline $\begin{array}{l}\text { Study } \\
\text { (Year) }\end{array}$ & Prediction Event & Method & Dataset & $\begin{array}{l}\text { Adopted } \\
\text { Features }\end{array}$ & AUC & Issue \\
\hline \multirow{2}{*}{$\begin{array}{c}{[52]} \\
(2009)\end{array}$} & $\begin{array}{l}\text { 30-day } \\
\text { pneumonia- } \\
\text { unrelated } \\
\text { readmission } \# 1\end{array}$ & \multirow{2}{*}{$\mathrm{LR}^{\# 2}$} & \multirow{2}{*}{$\begin{array}{c}1117 \text { pneumonia } \\
\text { patients } \\
\text { discharged at } \\
\text { Galdakao Hospital } \\
\text { in Basque country, } \\
\text { Spain }\end{array}$} & $\begin{array}{c}\text { Age, } \mathrm{CCI}^{\# 3} \text {, and } \\
\text { decompensated } \\
\text { comorbidities }\end{array}$ & 0.77 & \multirow{2}{*}{$\begin{array}{l}\text { The predictive } \\
\text { performances obtained } \\
\text { using only } 52 / 29 \\
\text { pneumonia- } \\
\text { unrelated/ } \\
\text { related readmission } \\
\text { cases were less } \\
\text { representative }\end{array}$} \\
\hline & $\begin{array}{l}\text { 30-day } \\
\text { pneumonia- } \\
\text { related } \\
\text { readmission } \# 1\end{array}$ & & & $\begin{array}{l}\text { Treatment failure } \\
\text { and instability } \\
\text { factors }\end{array}$ & 0.65 & \\
\hline $\begin{array}{l}{[53]} \\
2014\end{array}$ & $\begin{array}{l}\text { 30-day } \\
\text { all-cause } \\
\text { readmission }\end{array}$ & LR & $\begin{array}{l}965 \text { cases } \\
\text { (148 readmissions) } \\
\text { of pneumonia } \\
\text { admission } \\
\text { collected at } \\
\text { Hartford hospital, } \\
\text { Connecticut }\end{array}$ & $\begin{array}{l}16 \text { significant } \\
\text { features (5 } \\
\text { demographic } \\
\text { items, previous } \\
\text { admissions, } \\
\text { income, } \\
7 \text { comorbidities, } \\
\text { and } 2 \text { lab values) } \\
\text { selected from } \\
31 \text { variables }\end{array}$ & 0.71 & $\begin{array}{l}\text { Patients with age }< \\
65 \text { y were excluded }\end{array}$ \\
\hline $\begin{array}{l}{[54]} \\
2017\end{array}$ & $\begin{array}{l}\text { 30-day } \\
\text { all-cause } \\
\text { readmission }\end{array}$ & LR & $\begin{array}{l}\text { EHRs } \# 4 \text { of } 1463 \\
\text { patients (199 } \\
\text { readmissions) } \\
\text { hospitalized with } \\
\text { pneumonia } \\
\text { collected from } \\
6 \text { hospitals in } \\
\text { northern Texas }\end{array}$ & $\begin{array}{c}\text { Income, platelets, } \\
\text { prior } \\
\text { hospitalizations } \\
\text { in past year, vital } \\
\text { sign instabilities } \\
\# 5 \text { on discharge, } \\
\text { updated PSI } \# 6, \\
\text { and disposition } \\
\text { status at hospital } \\
\text { discharge }\end{array}$ & 0.731 & $\begin{array}{l}\text { Readmissions to } \\
\text { hospitals beyond } \\
\text { 100-mile radius of } \\
\text { Dallas were not } \\
\text { counted }\end{array}$ \\
\hline $\begin{array}{c}{[55]} \\
(2018)\end{array}$ & $\begin{array}{l}\text { 30-day } \\
\text { all-cause } \\
\text { readmission }\end{array}$ & LR & $\begin{array}{c}\text { EHRs of } 1295 \\
\text { hospitalizations } \\
\text { (330 readmissions) } \\
\text { with pneumonia at } \\
\text { the Cleveland clinic } \\
\text { main campus in } \\
\text { Ohio }\end{array}$ & $\begin{array}{c}13 \text { significant } \\
\text { features (age, } \\
\text { cancer, CHD } \# 7, \\
\text { stroke, antibiotics, } \\
\text { opioids, } \\
\text { temperature, } \\
\text { BUN } \# 8, \\
\text { hemoglobin, } \\
\text { albumin, sodium, } \\
\text { INR \#9, and prior } \\
\text { admissions } \\
\text { within } 6 \text { months) }\end{array}$ & 0.74 & $\begin{array}{l}\text { Excluded } \\
\text { age }<65 \text { y }\end{array}$ \\
\hline
\end{tabular}


Table 3. Cont.

\begin{tabular}{|c|c|c|c|c|c|c|}
\hline $\begin{array}{l}\text { Study } \\
\text { (Year) }\end{array}$ & Prediction Event & Method & Dataset & $\begin{array}{l}\text { Adopted } \\
\text { Features }\end{array}$ & AUC & Issue \\
\hline $\begin{array}{c}\text { Pilot study } \\
\text { [56] } \\
(2018)\end{array}$ & $\begin{array}{c}\text { 30-day } \\
\text { all-cause } \\
\text { readmission }\end{array}$ & IGS & $\begin{array}{l}1103 / 4331 \mathrm{w} / \text { wo } \\
\text { readmissions of } \\
\text { pneumonia } \\
\text { patients retrieved } \\
\text { from NHIRD } \\
\text { (medical } \\
\text { administrative } \\
\text { records) in } \\
\text { Taiwan }\end{array}$ & $\begin{array}{l}20 \text { features } \\
\text { (demographics, } \\
\text { comorbidity no., } \\
\text { comorbidity } \\
\text { index, events } \\
\text { within } 1 \text { year } \\
\text { before admission, } \\
\text { inpatient } \\
\text { interventions, } \\
\text { category of } \\
\text { admitted } \\
\text { hospitals, LoA } \\
\text { \#10, healthcare } \\
\text { cost, discharge } \\
\text { status, and } \\
\text { dosage of } \\
\text { antibiotics) }\end{array}$ & 0.76 & $\begin{array}{l}\text { Physiological signals, } \\
\text { laboratory test } \\
\text { results, and social } \\
\text { determinants, were not } \\
\text { included in NHIRD } \\
\text { and not adopted in our } \\
\text { pilot study }\end{array}$ \\
\hline This study & $\begin{array}{c}\text { 30-day } \\
\text { all-cause } \\
\text { readmission }\end{array}$ & $\begin{array}{c}\text { IGS, } \\
\text { DNN, and } \\
\text { LR }\end{array}$ & $\begin{array}{l}1545 / 6228 \mathrm{w} / \text { wo } \\
\text { readmissions of } \\
\text { pneumonia } \\
\text { patients retrieved } \\
\text { from NHIRD } \\
\text { (medical } \\
\text { administrative } \\
\text { records) in } \\
\text { Taiwan }\end{array}$ & $\begin{array}{l}49 \text { features listed } \\
\text { in Table } 4\end{array}$ & $\begin{array}{c}\mathbf{0 . 7 7 5 8} \\
0.7547, \\
\text { and } \\
0.7689\end{array}$ & $\begin{array}{l}\text { Physiological signals, } \\
\text { laboratory test } \\
\text { results, and social } \\
\text { determinants were not } \\
\text { included in NHIRD } \\
\text { and not adopted in } \\
\text { this study }\end{array}$ \\
\hline
\end{tabular}

\#1 of pneumonia patients; ${ }^{\# 2}$ logistic regression; ${ }^{\# 3} \mathrm{CCI}=$ Charlson comorbidity index; ${ }^{\# 4} \mathrm{EHR}=$ electronic health record; $\# 5$ vital sign instabilities were defined as temperature $\geq 37.8^{\circ} \mathrm{C}$, heart rate $>100$ beats $/ \mathrm{min}$, respiratory rate $>24$ breaths $/ \mathrm{min}$, systolic blood pressure $\leq 90 \mathrm{mmHg}$, or oxygen saturation $<90 \%$; $\# 6$ PSI = pneumonia severity index; ${ }^{\# 7} \mathrm{CHD}=$ coronary heart disease; ${ }^{\# 8} \mathrm{BUN}=$ blood urea nitrogen; ${ }^{\# 9} \mathrm{INR}=$ international normalized ratio; ${ }^{\# 10} \mathrm{LoA}=$ length of admission (days).

In [52], the model constructed for predicting pneumonia-unrelated readmissions of pneumonia admitted patients using logistic regression analysis by including data retrieved from the EHR of a single hospital achieved a predictive performance of AUC $=0.77$, while the AUC of the model constructed for predicting pneumonia-related readmission was only 0.65 .

In [53], a logistic model for predicting 30-day all-cause readmission was designed using a dataset extracted from the EHR of a tertiary-care hospital, reaching a predictive performance of AUC $=0.71$. The features included laboratory values, vital signs, age, sex, comorbidities, nursing home resident, marital status, income, prior admission, length of stay, etc. (Table 3). It suggested that income and number of previous admissions included for model construction significantly improved the predictive performance.

In [54], a full-stay model for predicting 30-day all-cause readmission was designed using the EHR data of 1463 pneumonia patients (13.6\% were readmitted) collected from six hospitals, including safety net, community, teaching, and nonteaching hospitals, achieving a predictive performance of AUC $=0.731$. The features selected for model construction included disposition status, vital sign instabilities on discharge, and an updated pneumonia severity index calculated using values from the day of discharge, etc. (Table 3). The full-stay pneumonia model (AUC $=0.731$ ) outperformed the first-day pneumonia model (AUC $=0.695$ ), Centers for Medicare and Medicaid Services pneumonia model $(\mathrm{AUC}=0.64)$, and two pneumonia severity scores (updated PSI: AUC $=0.673$ and CURB-65 score: $\mathrm{AUC}=0.604)$. 
Table 4. Comparisons between pneumonia patients with and without readmission.

\begin{tabular}{|c|c|c|c|}
\hline \multirow{2}{*}{ Variables } & \multicolumn{2}{|c|}{ Readmission } & \multirow{2}{*}{$p$-Value } \\
\hline & Yes $(n=1545)$ & No $(n=6228)$ & \\
\hline Gender $^{\mathrm{a}, \mathrm{b}, \mathrm{c}}, n(\%)$ & & & $<0.001$ \\
\hline Men & $1023(66.2 \%)$ & $3495(56.1 \%)$ & \\
\hline Women & $522(33.8 \%)$ & $2733(43.9 \%)$ & \\
\hline Age $^{\mathrm{b}}$ in year, mean (SD) & $74.7(15.1)$ & $65.7(20)$ & $<0.001$ \\
\hline \multicolumn{4}{|l|}{ Comorbidity, mean (SD) } \\
\hline No. ${ }^{a, b, c}$ & $3.6(0.9)$ & $2.8(1.4)$ & $<0.001$ \\
\hline CCI score & $2.2(1.9)$ & $0.9(1.3)$ & $<0.001$ \\
\hline \multicolumn{4}{|l|}{ Events within 1 year before admission } \\
\hline ED visits $\mathrm{b}, \mathrm{c}, n(\%)$ & $1224(79.2 \%)$ & $4621(74.2 \%)$ & $<0.001$ \\
\hline Hospitalizations a, b, c, mean (SD) & $2.2(1.9)$ & $1.5(1.1)$ & $<0.001$ \\
\hline Outpatient visits ${ }^{c}$, mean (SD) & $20.2(19.8)$ & $17(17.7)$ & $<0.001$ \\
\hline \multicolumn{4}{|l|}{ Inpatient Interventions } \\
\hline Surgical operations, mean (SD) & $1.1(1.4)$ & $0.7(1.1)$ & $<0.001$ \\
\hline Adm. Medications ${ }^{a}, b, c$, mean (SD) & $18.2(8.3)$ & $15(7.3)$ & $<0.001$ \\
\hline Ventilator use/therapy ${ }^{a}, b, c, n(\%)$ & $1149(74.4 \%)$ & $3650(58.6 \%)$ & $<0.001$ \\
\hline Other interventions $\mathrm{a}, \mathrm{b}, \mathrm{c}, n(\%)$ & $820(53.1 \%)$ & $1717(27.6 \%)$ & $<0.001$ \\
\hline Category of admitted hospitals $\mathrm{a}, \mathrm{b}, \mathrm{c}, n(\%)$ & & & $<0.01$ \\
\hline Medical center & $333(21.6 \%)$ & $1410(22.6 \%)$ & \\
\hline Regional hospital & $713(46.1 \%)$ & $3056(49.1 \%)$ & Chi-square =9.658; \\
\hline District hospital & $499(32.3 \%)$ & $1762(28.3 \%)$ & $p=0.008$ \\
\hline Length of admission ${ }^{b, c}$, mean (SD) days & $11.4(6.7)$ & $8.4(5.5)$ & $<0.001$ \\
\hline Total healthcare cost ${ }^{a}, b$, mean (SD) NT\$ & $54,268(46,311)$ & $36,975(39,346)$ & $<0.001$ \\
\hline Discharge status $b, c$ & & & 0.654 \\
\hline No follow-up, $n(\%)$ & $49(3.2 \%)$ & $184(3.0 \%)$ & \\
\hline Outpatient follow-up, $n(\%)$ & $1496(96.8 \%)$ & $6044(97.0 \%)$ & \\
\hline \multicolumn{4}{|c|}{ Outpatient visits within 1 year before admission, mean (SD) } \\
\hline Myocardial infarction $a, b, c$ & $0.2(2)$ & $0.2(1.9)$ & 0.594 \\
\hline Congestive heart failure & $2.5(7.8)$ & $1.7(6.2)$ & $<0.001$ \\
\hline Peripheral vascular disease ${ }^{b, c}$ & $0.4(2.7)$ & $0.3(2.4)$ & 0.128 \\
\hline Cerebrovascular disease ${ }^{b}$ & $8(18.1)$ & $6.3(17.3)$ & 0.001 \\
\hline Dementia $^{a}$ & $2.9(8.4)$ & $2.3(8.1)$ & 0.008 \\
\hline Chronic pulmonary disease ${ }^{b}$ & $0.2(2.2)$ & $0.1(2.1)$ & 0.408 \\
\hline Rheumatologic disease ${ }^{a, c}$ & $0.5(5.6)$ & $0.5(4.9)$ & 0.833 \\
\hline Peptic ulcer disease ${ }^{a, b, c}$ & $2.4(6.3)$ & $2.1(6.1)$ & 0.069 \\
\hline Mild liver disease $^{a}$ & $1.3(6.4)$ & $1.3(5.9)$ & 0.815 \\
\hline Diabetes w/o chron. compl. & $6.1(13.8)$ & $5.6(13.1)$ & 0.214 \\
\hline Diabetes w chron. compl. ${ }^{a, b, c}$ & $1.1(4.9)$ & $1.3(6)$ & 0.25 \\
\hline Hemiplegia or paraplegia a, c & $0.7(6.7)$ & $0.6(5.4)$ & 0.334 \\
\hline Renal disease ${ }^{a, b, c}$ & $4.1(14.3)$ & $3.6(14.6)$ & 0.283 \\
\hline Leukemia or lymphoma $a, b, c$ & $5.6(15.5)$ & $3.6(14.4)$ & $<0.001$ \\
\hline Moderate/severe liver disease $b, c$ & $0(0.6)$ & $0(0.6)$ & 0.668 \\
\hline Metastatic solid tumor & $0.2(2.9)$ & $0.1(2)$ & 0.069 \\
\hline AIDS/HIV b, c & $0.1(1.7)$ & $0(0.9)$ & 0.298 \\
\hline \multicolumn{4}{|c|}{ Hospitalizations within 1 year before admission, mean (SD) } \\
\hline Myocardial infarction $\mathrm{a}, \mathrm{b}, \mathrm{c}$ & $0.1(0.3)$ & $0(0.2)$ & $<0.001$ \\
\hline Congestive heart failure $a, b, c$ & $0.6(1.4)$ & $0.2(0.9)$ & $<0.001$ \\
\hline Peripheral vascular disease ${ }^{a, b, c}$ & $0.1(0.3)$ & $0(0.2)$ & $<0.001$ \\
\hline Cerebrovascular disease ${ }^{a, b, c}$ & $0.6(1.4)$ & $0.3(1)$ & $<0.001$ \\
\hline Dementia ${ }^{b, c}$ & $0.1(0.6)$ & $0.1(0.4)$ & $<0.001$ \\
\hline Chronic pulmonary disease ${ }^{\mathrm{a}, \mathrm{b}}$ & $0(0.3)$ & $0(0.2)$ & 0.052 \\
\hline Rheumatologic disease $\mathrm{b}, \mathrm{c}$ & $0.1(0.7)$ & $0(0.3)$ & 0.007 \\
\hline Peptic ulcer disease ${ }^{a, b, c}$ & $0.3(0.8)$ & $0.1(0.5)$ & $<0.001$ \\
\hline
\end{tabular}


Table 4. Cont.

\begin{tabular}{|c|c|c|c|}
\hline \multirow{2}{*}{ Variables } & \multicolumn{2}{|c|}{ Readmission } & \multirow{2}{*}{$p$-Value } \\
\hline & Yes $(n=1545)$ & No $(n=6228)$ & \\
\hline Mild liver disease $^{c}$ & $0.3(1.3)$ & $0.1(0.6)$ & $<0.001$ \\
\hline Diabetes w/o chron. compl. a, b & $1.1(2.3)$ & $0.5(1.2)$ & $<0.001$ \\
\hline Diabetes w chron. compl. a, c & $0.1(0.7)$ & $0.1(0.4)$ & $<0.001$ \\
\hline Hemiplegia or paraplegia b, c & $0.1(0.7)$ & $0(0.3)$ & $<0.001$ \\
\hline Renal disease ${ }^{a, b, c}$ & $0.5(1.7)$ & $0.2(1)$ & $<0.001$ \\
\hline Leukemia or lymphoma ${ }^{a, b, c}$ & $1(2.9)$ & $0.3(1.1)$ & $<0.001$ \\
\hline Moderate/severe liver disease ${ }^{a, b, c}$ & $0.1(0.7)$ & $0(0.2)$ & $<0.001$ \\
\hline Metastatic solid tumor ${ }^{b}$ & $0.5(2)$ & $0.1(0.6)$ & $<0.001$ \\
\hline AIDS/HIV b, c & $0(0.2)$ & $0(0.1)$ & 0.043 \\
\hline
\end{tabular}

Note: Variables selected by IGS models with ${ }^{\mathrm{a}} O B 1,{ }^{\mathrm{b}} O B 2$, and ${ }^{\mathrm{c}} O B 3$, respectively.

In [55], a logistic model was created using an EHR dataset of 1295 pneumonia patients, in which 330 patients were readmitted within 30 days after discharge, with a readmission rate as high as $25.5 \%$. A total of 13 features were adopted for the model design, in which linear or nonlinear relationship fitting between the continuous features and readmission outcome were obtained, and it achieved a predictive performance of $\mathrm{AUC}=0.74$.

\subsection{Problem Statements and Research Objectives}

As the readmission rate is deemed as an indicator of hospital healthcare quality [4], HRRP has been adopted to improve healthcare quality, reduce healthcare costs, and elevate patient satisfaction [6]. A predictive model designed to identify patients with a higher readmission probability is useful for post-discharge interventions to save aftercare cost; for example, identifying COPD patients with a high potential readmission for performing readmission mitigation interventions saved $90 \%$ of the healthcare cost when compared to patients provided with costly aftercare [9]. Hence, designing models to precisely predict high-risk readmissions patients admitted with pneumonia for post-discharge intervention is crucial for the prevention of patient readmissions and for decreasing the healthcare cost. However, most models for predicting the readmission of discharged pneumonia patients performed poorly, with AUCs ranging from 0.71 to 0.74 [53,55], and they were mostly designed based on data collected from a single medical center or were validated among older patients by excluding patients younger than 65 years old [53,55]. Although, in certain healthcare settings, these models might be useful in readmission prediction, we still need to enhance the predictive performance for admitted pneumonia patients caused by non-typical influenza, such as SARS, MERS, or COVID-19, which become more and more widespread.

In our previous report [56], we designed models for predicting readmissions for patients admitted with pneumonia by applying the IGS (integrated genetic algorithm with support vector machine) algorithm based on the data retrieved from the National Health Insurance Research Database (NHIRD) with 20 features adopted, and we achieved a predictive performance with an accuracy, sensitivity, specificity, and AUC of $69.33-71.44 \%$, $66.27-69.41 \%, 69.32-72.24 \%$, and $0.7518-0.7601$, respectively. In the current study, our objective was to design predictive models using NHIRD with more features (49 features) to further improve the predictive performance in the identification of high-risk readmission patients admitted with pneumonia. Moreover, in contrast to data collected from a single hospital for model design, as reported in previous studies [53,55], NHIRD covers multiple hospital data submitted by more than $97 \%$ of hospitals scattered around different areas in Taiwan for claiming healthcare reimbursement from the Bureau of National Health Insurance. Additionally, deep neuro network (DNN) and logistic regression (LR) were also applied to design the predictive models, so as to be compared with the models created using the IGS algorithm. 


\section{Materials and Methods}

\subsection{Data Source}

The data were retrieved from a subset including the claim data of 1 million patients randomly sampled from the NHIRD, containing information of medical facility registries, inpatient orders, ambulatory care, prescription drugs, and physicians providing services to the entire 23 million Taiwanese population enrolled in the NHI program. The diagnoses in the NHIRD dataset were coded according to the International Classification of Diseases, ninth edition, Clinical Modification (ICD-9-CM), and the dataset has been widely used for studying issues of public health and the causal relationship of a disease associated with other comorbidities [57].

\subsection{Samples}

Data of patients older than 20 years old admitted within 2010-2011 due to pneumonia (ICD Code 480.xx, 481, 482.xx, 483.0, 483.x, 485, 486, and 487.0) were retrieved from the NHIRD for constructing the predictive models. Patient claim data collected in 2010 were used for the training the models, while those collected in 2011 were used for the testing. The training and testing datasets included 3911 (761 readmissions and 3150 non-readmissions) and 3862 patient data (784 readmissions and 3078 non-readmissions), respectively, showing that the datasets were highly imbalanced, with the ratio of the majority to minority samples reaching 4.14 and 3.93, respectively. The readmitted patients were those who had been admitted again with all-cause conditions within 30 days after being discharged from the hospital. Table 4 compares the included variables between readmitted and nonreadmitted patients of the combined training and testing datasets (1545 readmissions and 6228 non-readmissions).

When classifying the imbalanced dataset, it has to be noted that samples in the majority class outnumbered those in the minority class, which is often of more interest or importance, making the algorithms optimized with accuracy adopted as the objective function biased toward the majority class [58]. In general, the accuracies of these models are satisfactory, yet their sensitivities are quite low. Hence, alternative fitness functions, such as AUC [59] or weighted sum of accuracy, sensitivity, and specificity [60], have been proposed to solve this problems.

\subsection{Variables}

To design the predictive models, a total of 49 candidate variables were considered, including gender; age; comorbidity number and comorbidity index, i.e., Charlson comorbidity index (CCI) [61]; medical events (ED visits, hospitalizations, and outpatient visits) within 1 year before admissions; inpatient interventions, number of surgical operations, number of administrated medications, ventilator use/therapy (ventilator therapy, oxygen inhalation, humidity inhalation, or vapor/aerosol therapy), and other accompanied therapies (urinal indwelling, C.V.P. catheter, N-G feeding, respiratory suction, or tracheostomy care); category of admitted hospitals (medical center, regional hospital, or district hospital); length of admission; total healthcare cost; discharge status; diagnosed comorbidities included within CCI in outpatient visits; and hospitalizations within 1 year before pneumonia admission.

\subsection{Statistica Analysis}

The statistic tool (SPSS 22.0, IBM) was adopted for the descriptive and inferential analyses. Distribution differences in the demographic characteristics, events within 1 year before admission, inpatient interventions, category of admitted hospitals, and discharge status of readmitted and non-readmitted patients were compared using the Chi-square test. Difference in continuous variables were compared with the unpaired Student's $t$-test. The statistical significance was defined as $p<0.05$. 


\subsection{Design of Prediction Models}

Figure 1 shows the experimental procedure with IGS, DNN, and LR algorithms used for creating the predictive models. Figure 2 shows the procedures of IGS, DNN, and LR algorithms for optimizing the predictive performances of the models. As illustrated in Figure 2a, in the IGS algorithm, GA was used for selecting the salient features and adjusting the SVM parameters (cost value and kernel parameter), whereas SVM was used for designing the predictive models based on three different objective functions [60]. For each iteration when optimizing the IGS model, the $n$ chromosomes were updated by combining $n / 2$ new chromosomes generated from crossover with the other $n / 2$ chromosomes obtained from mutation. The aforementioned steps were repeated until the best objective value was obtained within the maximum number of iterations. As illustrated in Figure $2 b, c$, hyperparameter tuning adopting the GridSearchCV function was used for optimizing the LR and DNN models, respectively.

Model Training: 3911 samples (761 readmissions and 3150 nonreadmissions), each consisting of 49 variables

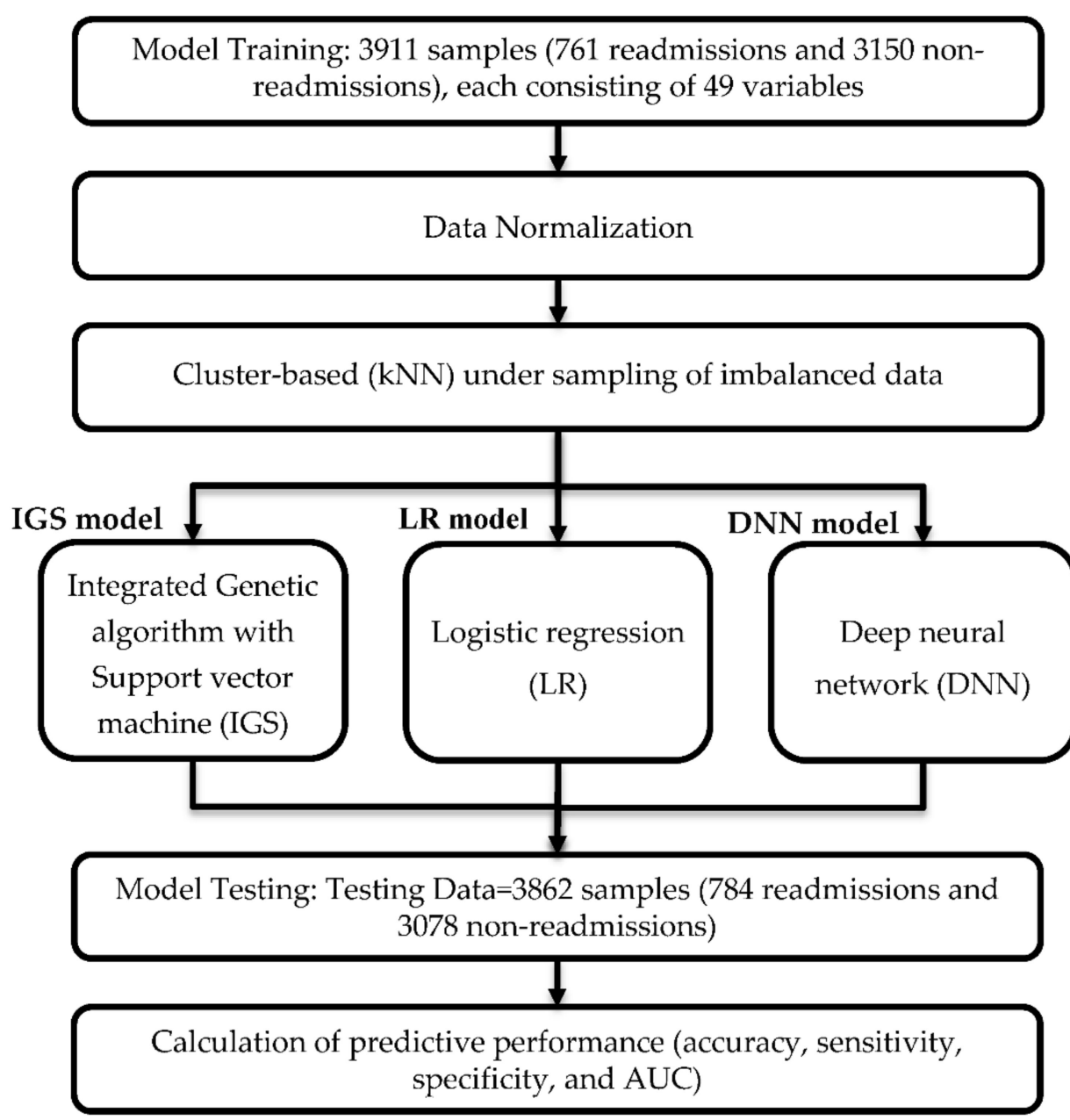

Figure 1. Experimental procedure for designing IGS, DNN, and LR predictive models.

In the training phase, the cluster-based $\mathrm{kNN}$ ( $k$-nearest neighbors) undersampling method [62] was adopted to prepare the training dataset. The samples in the majority class (with $M$ samples) were clustered into $m$ clusters that each consisted of $M / m$ or $M / m+1$ samples. Then, the kNN algorithm was applied to select the sample that was nearest to the center of gravity in each cluster, resulting in a balanced training set containing $2 m$ samples ( $m$ samples obtained from the majority group and $m$ samples of minority group) for cross validation. Ten-fold cross validation was adopted for training and validating the models in order to obtain a model with the best performance. In the testing phase, the imbalanced 
testing dataset was applied to test the constructed predictive models obtained in the training phase.

(a) IGS algorithm

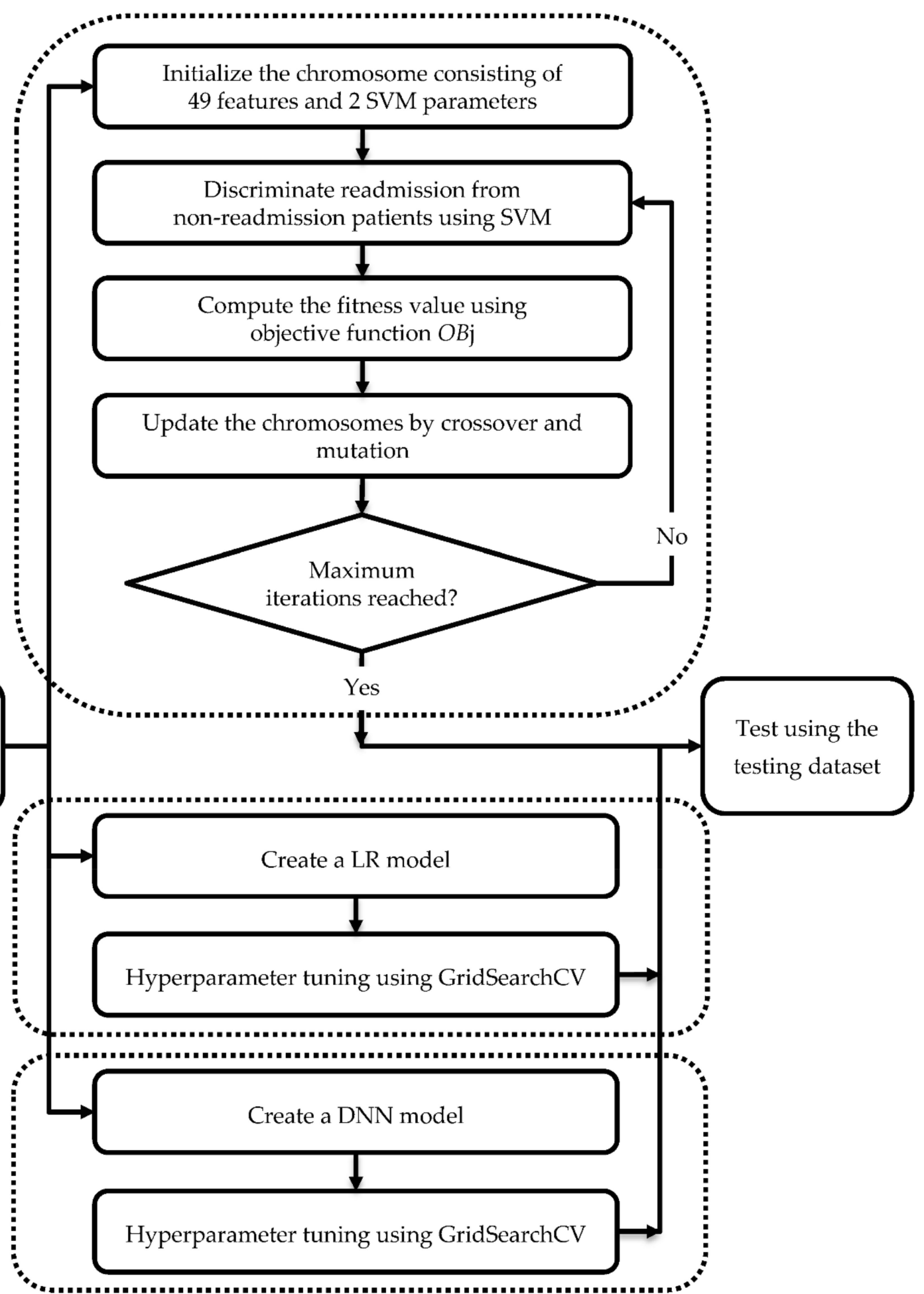

Figure 2. Optimization procedures of (a) IGS, (b) LR, and (c) DNN algorithms.

When designing the IGS models, selection of the objective function was crucial for obtaining the optimal models. As indicated in Equations (1)-(3), in this study, the cost- 
sensitive objective functions, including combined accuracy, sensitivity, and specificity, AUC, as well as g-mean, were used to obtain the optimal models with imbalanced datasets.

$$
\begin{gathered}
\mathrm{OB} 1=\mathrm{ACC}-|\mathrm{SEN}-\mathrm{SPE}| \\
\mathrm{OB} 2=\mathrm{AUC} \\
\mathrm{OB} 3=\sqrt{\mathrm{SEN} \times \mathrm{SPE}}
\end{gathered}
$$

Notice that in Equation (1), the maximum fitness value is obtained by maximizing accuracy (ACC) and minimizing difference between sensitivity (SEN) and specificity (SPE) to avoid the decision hyperplane biasing toward the majority class. In the testing phase, the testing dataset was adopted for testing the models created in the training phase. Performances of the constructed models were quantitatively evaluated using the SEN, SPE, ACC, and AUC.

The IGS models were developed using the SVM package (LIBSVM $[63,64])$ and GA algorithms under a Visual C++ environment operated under a Windows 10 operating system in a personal computer with Intel i7-6700HQ 4-core CPU @2.60 GHz, 8 GB main memory, and NVidia GeForce GTX950M GPU. As illustrated in Figure 3, the structure of the DNN algorithm consisted of an input layer, three hidden layers, and an output layer with 49, 20-20-20, and 1 nodes, respectively, with a rectified linear (ReLU) function adopted in the hidden layer and sigmoid activation function applied in the output layer. In model training, the epoch was set to 80 and the batch size was set to 50. A personal computer consisted of Intel i7-7500U dual-core CPU @2.70 GHz, 8 GB main memory, and NVidia Geforce MX150 GPU and was operated under a Windows 10 operating system, and the Jupyter Notebook (Scikit-learn package, Tensorflow, Python) environment was used for designing the LR and DNN models [65-67].

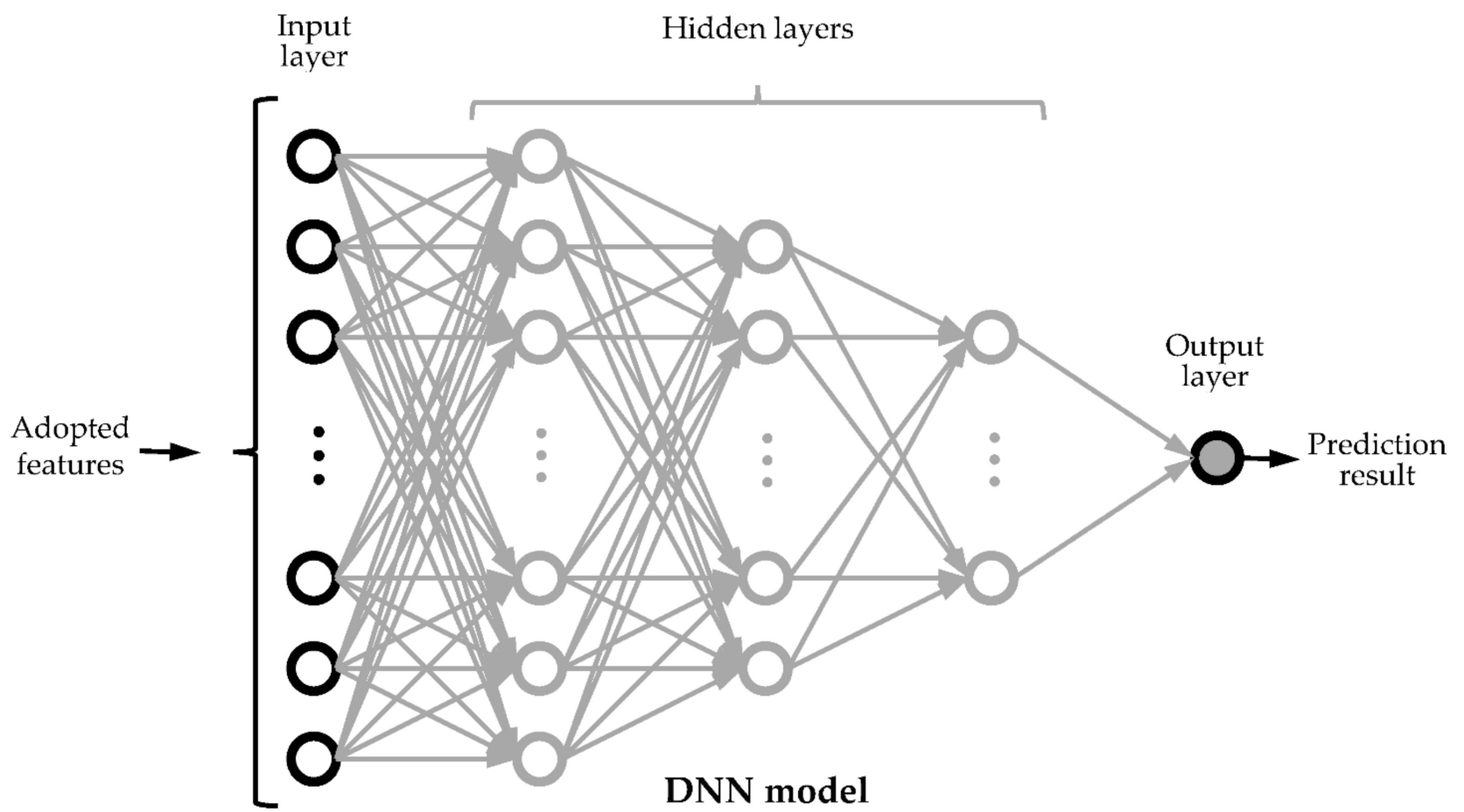

Figure 3. Structure of deep neural network (DNN). 


\section{Results}

3.1. Comparisons of Demographic Characteristics, Comorbidities, Inpatient Interventions, and Related Variables between Readmitted and Non-Readmitted Patients

As shown in Table 4, data of readmitted (1545 patients; 1023 men and 522 women) and non-readmitted (6228 patients; 3495 men and 2733 women) patients retrieved from the NHIRD within 2010-2011 were compared. As indicated in the table, the age and gender distribution of the readmitted and non-readmitted patients were significantly different $(p<0.001)$. Readmitted patients were older and mostly male. Regarding comorbidity, readmitted patients had significantly more comorbidities $(p<0.001)$ and higher CCI $(p<0.001)$ scores than the non-readmitted patients. It was also noted that readmitted patients experienced significantly more ED visits $(p<0.001)$ and exhibited a significantly higher frequency of hospitalizations $(p<0.001)$ and outpatient visits $(p<0.001)$ within 1 year before admission.

During hospitalization, readmitted patients received significantly more surgical operations and administrated medications $(p<0.001)$ and also had a significantly higher chance of using a ventilator and receiving additional interventions $(p<0.001)$, including urinal indwelling, C.V.P. catheter, N-G feeding, respiratory suction, or tracheostomy care. In addition, readmitted patients had longer hospital stays and more healthcare expenditure than the non-readmitted patients $(p<0.001)$.

The category of admitted hospitals between readmitted and non-readmitted groups was significantly different $(p<0.01)$. The admission rate to district hospitals for the readmitted group was higher than the non-readmitted group. There was no significant difference ( $p=0.654$ ) regarding discharge status; the outpatient follow-up rate of the readmitted group was similar to the non-readmission group.

When comparing hospitalizations within 1 year before admission for 17 comorbidities, except chronic pulmonary disease (marginally significant with $p=0.052$ ), the frequencies of all the other comorbidities in the admitted group were significantly higher $(p<0.05)$ than the non-readmitted group. In contrast, only four comorbidities, including congestive heart failure, cerebrovascular disease, dementia, and blood malignancy (leukemia or lymphoma), in the readmitted group exhibited a significant higher frequency of outpatient visits than the non-admitted group.

\subsection{Predictve Performance}

Table 5 compares the predictive performance among five different models designed using IGS, DNN, and LR algorithms, with the training dataset prepared using a clusterbased undersampling method $(\mathrm{kNN})$. Accuracy was calculated as the fitness value for DNN and LR models, while three objective functions were applied for optimizing the IGS models. As shown in Table 5, the predictive performance of the DNN and LR models achieved $61.50 \%, 79.34 \%, 56.95 \%$, and 0.7547 , as well as $65.77 \%, 78.44 \%, 62.54 \%$, and 0.7689 in ACC, SEN, SPE, and AUC, respectively. The IGS models using AUC as the objective function exhibited a better predictive performance than the other models with ACC, SEN, SPE, and AUC achieving 70.30-71.22\%, 68.85-78.33\%, 70.30-73.58\%, and 0.7536-0.7729, respectively, in the training phase, as well as 68.20-70.11\%, 70.40-74.61\%, 66.56-69.59\%, and $0.7599-0.7758$, respectively, in the testing phase.

As indicated in Table 5, although the model trained using OB2 achieved a slightly better predictive performance, the IGS models optimized with objective functions OB1, $\mathrm{OB} 2$, and $\mathrm{OB} 3 \mathrm{had}$ a similar predictive performance in both the training and testing phases. The ACC, SEN, SPE, and AUC obtained in the testing phase were $68.20 \%, 74.61 \%, 66.56 \%$, and 0.7727 , respectively, for the IGS-OB1 model; 70.11\%, 73.46\%, 69.26\%, and 0.7758 , respectively, for IGS-OB2 model; and $69.75 \%, 70.40 \%, 69.59 \%$, and 0.7599 , respectively, for the IGS-OB3 model. The variables selected by the IGS models optimized with three objective functions were different (Table 4). The salient variables selected by the IGS method for designing the predictive models included gender, age, number of comorbidities, ED visits, frequency of hospitalizations and outpatient visits within 1 year before admission, 
number of administrated medications, ventilator use and accompanied therapies, other interventions, category of admitted hospital, length of admission, total healthcare cost, discharge status, and outpatient visits and hospitalizations visits for comorbidity within 1 year before admission.

Table 5. Predictive performances of models designed by the IGS, DNN, and LR methods.

\begin{tabular}{|c|c|c|c|c|c|}
\hline Method & $\begin{array}{l}\text { Objective } \\
\text { Function }\end{array}$ & $\operatorname{ACC}(\%)$ & SEN (\%) & SPE (\%) & AUC \\
\hline \multicolumn{6}{|c|}{ Training Phase } \\
\hline \multirow{3}{*}{ IGS } & OB1 & 70.30 & 70.30 & 70.30 & 0.7536 \\
\hline & OB2 & 70.63 & 78.33 & 72.93 & 0.7729 \\
\hline & OB3 & 71.22 & 68.85 & 73.58 & 0.7595 \\
\hline DNN & $\mathrm{ACC}$ & 65.81 & 65.04 & 66.54 & 0.7266 \\
\hline LR & $\mathrm{ACC}$ & 69.18 & 65.45 & 72.76 & 0.7543 \\
\hline \multicolumn{6}{|c|}{ Testing Phase } \\
\hline \multirow{3}{*}{ IGS } & OB1 & 68.20 & 74.61 & 66.56 & 0.7727 \\
\hline & OB2 & 70.11 & 73.46 & 69.26 & 0.7758 \\
\hline & OB3 & 69.75 & 70.40 & 69.59 & 0.7599 \\
\hline DNN & $\mathrm{ACC}$ & 61.50 & 79.34 & 56.95 & 0.7547 \\
\hline LR & $\mathrm{ACC}$ & 65.77 & 78.44 & 62.54 & 0.7689 \\
\hline
\end{tabular}

The execution time was $70.2 \mathrm{~s}$ and $1.8 \mathrm{~s}$ for training and testing the DNN models, respectively, and $2.2 \mathrm{~s}$ and $1.6 \mathrm{~s}$ for training and testing the LR models, respectively. The time for training the IGS model with each objective function was much longer (around 1-2 weeks) compared to the LR and DNN models, while the time for testing the IGS model was around a few seconds only.

\section{Discussions}

\subsection{Model Explainability}

As shown in Table 4, among the 49 variables included for analysis, 34 showed significant differences $(p<0.05)$ between readmitted and non-readmitted patients. Variables including gender; comorbidity number; hospitalization number within 1 year before admission; adminstrated medication number and ventilator use/therpies, and other interventions; catergery of admitted hospitals; mean outpatient visits within 1 year before admission for myocardial infarction, peptic ulcer disease, diabetes with chronic complication, renal disease, and leukemia/lymphoma; and mean hospitalization within 1 year before admission for myocardial infarction, congestive heart failure, peripheral vascular disease, cerebrovascular disease, peptic ulcer disease, renal disease, leukemia/lymphoma, and moderate/severe liver disease were all selected by the three IGS models. Although highly statistically significant in discriminating the readmitted patients from the non-readmitted ones, variables, including age $(p<0.001)$; all-cause $(p<0.001)$, cerebrovascular disease $(p<0.01)$, and dementia $(p<0.01)$ outpatient visits within 1 year before admission; and mild liver disease $(p<0.001)$ and metastatic solid tumor $(p<0.001)$ hospitalizations within 1 year before admission, were selected by only one IGS model. On the other hand, although not reaching statistical significance, discharge status $(p=0.654)$ and outpatient visits within 1 year before admission for myocardial infarction $(p=0.594)$, peripheral vascular disease $(0.128)$, chronic pulmonary disease $(0.408)$, rheumatologic disease $(0.833)$, peptic ulcer disease (0.069), mild liver disease (0.815), diabetes with chronic complication (0.25), hemiplegia/paraplegia (0.334), renal disease (0.283), moderate/severe liver disease (0.668), and AIDS/HIV (0.298) were selected by at least one IGS model. These findings verified that filter methods, such as statistical analysis, F-score, or entropy, may not be appropriate for selecting features when designing predictive models $[60,64]$. Moreover, it also revealed that non-significant variables observed by univariate statistical analysis may have compensatory 
effects for increasing the predictive performance of the created models. Variables that are highly correlated can also be adopted together to strengthen the predictive performances of the predictive models [64].

As indicated in Table 5, the AI model with the best predictive performance in the testing phase was the IGS model optimized with the OB2 objective function. As indicated in Table 4, the number of men was more than women in the readmission group, which is consistent with previous studies [21,22], but inconsistent with another study regarding pneumonia readmission [54]. The age of readmission group was significantly older than the non-readmission group, which is consistent with previous studies [21,54], indicating that the elder patients usually have worse health status and thus have a higher risk of readmission than the younger patients.

The number of comorbidities in the readmission group was significantly more than that of the non-readmission group, denoting that pneumonia patients with more comorbidities had a higher risk of readmission after discharge, which is consistent with a previous study [21]. Frequencies of ED visits and hospitalizations within 1 year before admission were both significantly more in the readmitted group than the non-readmitted group, which is consistent with previous studies [53-55], reinforcing that pneumonia patients with more recent ED visits or hospitalizations exhibited worse health status and thus had a higher risk of readmission after discharge.

During hospitalization, pneumonia patients received more administrated medications and also had higher rates of using ventilators and receiving additional interventions, again indicating that readmitted patients tended to have worse health status, resulting in a higher chance of readmission after discharge. The above inpatient intervention variables were adopted for designing our models, but not in the previous study [55]. In [55], only administrated opioids and time until first administered antibiotics were included. Notice that, as shown in Table 4, the number of surgical operations was not selected by any IGS models, even it was significantly different between the readmitted and non-readmitted groups.

Length of admission and total healthcare cost were both selected by the IGS-OB2 model and were also adopted in [44]. Pneumonia patients who stayed longer in hospital and accumulated higher medical expenses tended to be more severe and had a higher chance of future readmissions. Interestingly, for readmitted patients, only 4 among 17 comorbidities treated in outpatient visits within 1 year before admission were significantly more frequent $(p<0.05)$ than the non-readmitted patients, indicating that patients with frequent physician visit had better management of comorbidities and had the effect of preventing the deterioration of comorbidities [68], which in turn presented a lower chance of readmission. In contrast, 16 (and 1 marginally significant $p=0.052$ for CPD) out of 17 comorbidities causing hospitalizations within 1 year before admission for the readmitted patients exhibited significantly higher rates than the non-readmitted patients, denoting that health status presented in recent hospital admissions, especially the last one, was useful for predicting the following readmission. Trajectory of previous admissions and prior readmissions were the most important features for precisely discriminating readmission from non-readmission patients [44].

\subsection{Performance Comparison}

Table 3 compares the state-of-the-art models for predicting the readmission of pneumonia patients after discharge. It can be observed that many previous works adopted logistic regression (LR) with different features extracted from EHRs for designing the predictive models. The IGS models presented in this study achieved an AUC of 0.7727-0.7758, which is similar to the model reported in [52] (0.77) and outperforms the models presented in [53] (0.71), [54] (0.731), and [55] (0.74), which are designed based on EHRs. In [52], the AUC of LR models for predicting 30-day pneumonia-unrelated and pneumonia-related readmissions for patients with pneumonia admission reached 0.77 and 0.65 , respectively, in which only 52 cases of pneumonia-unrelated readmission and 29 pneumonia-related read- 
mission were observed among the 1117 pneumonia admitted patients who were included for model construction.

On the other hand, in $[53,55]$, younger cases $<65$ y old were excluded from the extracted samples when designing the predictive models. In contrast, our study included patients $\geq 20$ y old for the model design, and was more comprehensive and representative for readmission prediction. As demonstrated in Table 4, in general, elder patients had a significantly higher chance of readmission compared to the younger patients. In Taiwan, the NHI program provides healthcare to more than $99 \%$ of the $23 \mathrm{M}$ citizens. NHIRD has collected more than 20 years of long-term patient data for almost all Taiwanese citizens from the data submitted by the participating healthcare institutions for claiming reimbursements. Most citizens intend to migrate around hospitals to visit physicians with a high reputation for better treatment due to the good accessibility, comprehensive population coverage, short waiting time, and low costs, making the long-term collection of healthcare data for an individual patient in a single hospital very difficult. The healthcare data of an individual are generally distributed in the EHRs of many hospitals situated in different areas of Taiwan. Therefore, in general, EHRs have recorded only incomplete or short-term patient healthcare data [22]. In this study, compared to the model constructed with EHRs, our models were designed based on the long-term NHIRD dataset, which is capable of exhibiting the longterm health status of a patient, resulting in an improved predictive performance.

The TADEL method reached an excellent predictive performance with an AUC of 0.884 for predicting the all-cause readmission for all-cause conditions, with the performance improvement mainly achieved by adopting an attenuation coefficient and amplification coefficient [44]. However, in [44], the ratios of the readmission group to the non-readmission group in numbers of admissions and ED visits were 16.3 and 16.8, respectively, which were very high compared to our study (admission: 1.5; ED visit: 1.1) and a recent study (admission: 1.6) for predicting all-cause readmission [21]. We suggest that data with such a high ratio of readmission to non-readmission groups in the number of hospitalizations and ED visits presented in [44] could enable the designed model to achieve a higher predictive performance. Furthermore, a balanced dataset was adopted for designing the TADEL model in [44]; in contrast, our model and other models reported in previous studies were designed based on the imbalanced dataset, more similar to the real world, with a ratio of readmission to non-readmission case number of 4 in our model, 13.8 in [52], 6.5 in [53], 7.4 in [54], and 3.9 in [55], which greatly degraded the predictive performance of the created models.

As demonstrated in [69], laboratory test results, including the white blood cell count and albumin at discharge, as well as the number of comorbidities, are independent risk factors of readmission for pneumonia patients. However, compared to the predictive models presented in [54,55], variables associated with laboratory test results (concentration of platelets, albumin, and blood urea nitrogen) were not used in our models, as they were not available in the NHIRD. Although not significant $(p=0.654)$ in the discrimination readmitted from non-readmitted patients, discharge status (with/without outpatient followup) was selected by constructing two IGS models. As noted in [70], discharge disposition was an independent predictor of readmission for community-acquired pneumonia patients, and follow-up interventions after patient discharge is necessary for reducing morbidities and mortalities.

Variables that were used for creating the predictive models in this study and also adopted in previous studies include age, gender, CCI, LOS, previous admissions, and disposition status at hospital discharge, as well as related comorbidities, including congestive heart failure, coronary heart disease, cerebrovascular disease, chronic lung disease, renal disease, diabetes mellitus, and major psychiatric disorders [52-55].

\subsection{Future Works}

Only a moderate predictive performance (AUC < 0.8) has been achieved so far for models designed for predicting the readmission of pneumonia admission. In contrast, models 
for predicting all-cause readmissions of discharged patients admitted with all-cause conditions exhibited a higher predictive performance with an AUC as high as 0.877-0.9038 [46]. As shown in Table 4, the laboratory test results, physiological parameters, and social determinants of health available in EHRs were not included in the NHIRD dataset and were not available for our model design. Future work will focus on applying transfer learning algorithm [71-73] to transfer the knowledge learned from models obtained using the long-term NHIRD dataset to train models based on the short-term EHRs for improving the predictive performance.

In addition to transfer learning, other advanced AI methods, such as extreme gradient boosting (XGBoost) [37-39], time trajectory learning of adopted features [44], integrated AI models [45], feature selection algorithms [46,47], and graph-based methods [48] mentioned in Section 1.3, may also be adopted for model construction to further elevate the predictive performance of pneumonia readmission prediction.

\section{Conclusions}

According to the analytical results, it was observed that the IGS model for predicting pneumonia readmissions designed using NHIRD outperformed the models designed using data retrieved from EHRs presented in previous studies. It could be adopted to assist physicians in detecting pneumonia patients who have a higher risk of readmission after being discharged so that appropriate aftercare interventions can be administrated to prevent readmissions in order to reduce morbidities, mortalities, and healthcare cost. Future research will focus on further improving the predictive performance by using advanced AI methods as well as more significant features.

Author Contributions: Conceptualization, D.-J.L. and Y.-F.C.; Data curation, H.-H.L. and C.-S.L.; Formal analysis, J.-C.H. and H.-H.L.; Funding acquisition, F.-H.W. and Y.-F.C.; Investigation, F.-H.W. and C.-S.L.; Methodology, J.-C.H., F.-H.W. and H.-H.L.; Project administration, Y.-F.C.; Resources, J.-C.H. and C.-S.L.; Software, J.-C.H. and H.-H.L.; Supervision, D.-J.L. and Y.-F.C.; Writing-original draft, F.-H.W.; Writing — review \& editing, F.-H.W. and Y.-F.C. All authors have read and agreed to the published version of the manuscript.

Funding: This research was funded by Ministry of Science and Technology, Taiwan (grant nos. MOST109-2410-H-166-001 and MOST110-2410-H-166-001-SSS) and China Medical University, Taiwan (grant no. CMU110-N-07).

Institutional Review Board Statement: This study was approved by the Institute of Reviewing Board (No. 109-32) of Jen-Ai Hospital, Taichung, Taiwan.

Acknowledgments: The authors would like to express their appreciation to Ministry of Science and Technology, Taiwan and China Medical University, Taiwan for financial supports to Y.F. Chen and F.H. Wu, respectively.

Conflicts of Interest: The authors declare no conflict of interest.

\section{References}

1. Kansagara, D.; Englander, H.; Salanitro, A.; Kagen, D.; Theobald, C.; Freeman, M.; Kripalani, S. Risk prediction models for hospital readmission: A systematic review. JAMA 2011, 306, 1688-1698. [CrossRef] [PubMed]

2. Navathe, A.S.; Zhong, F.; Lei, V.J.; Chang, F.Y.; Sordo, M.; Topaz, M.; Navathe, S.B.; Rocha, R.A.; Zhou, L. Hospital readmission and social risk factors identified from physician notes. Health Serv. Res. 2018, 53, 1110-1136. [CrossRef] [PubMed]

3. Glans, M.; Ekstam, A.K.; Jakobsson, U.; Bondesson, Å.; Midlöv, P. Risk factors for hospital readmission in older adults within 30 days of discharge-A comparative retrospective study. BMC Geriatr. 2020, 20, 467. [CrossRef] [PubMed]

4. Dharmarajan, K.; Hsieh, A.F.; Lin, Z.; Bueno, H.; Ross, J.S.; Horwitz, L.I.; Barreto-Filho, J.A.; Kim, N.; Suter, L.G.; Bernheim, S.M. Hospital readmission performance and patterns of readmission: Retrospective cohort study of Medicare admissions. BMJ 2013, 347, f6571. [CrossRef]

5. Rumball-Smith, J.; Blakely, T.; Sarfati, D.; Hider, P. The mismeasurement of quality by readmission rate: How blunt is too blunt an instrument? A quantitative bias analysis. Med. Care 2013, 51, 418-424. [CrossRef]

6. Ayabakan, S.; Bardhan, I.; Zheng, Z. Triple Aim and the Hospital Readmission Reduction Program. Health Serv. Res. Manag. Epidemiol. 2021, 8, 2333392821993704. [CrossRef] 
7. Hoffman, G.J.; Yakusheva, O. Association between financial incentives in Medicare's hospital readmissions reduction program and hospital readmission performance. JAMA Netw. Open 2020, 3, e202044. [CrossRef]

8. Hogan, T.H.; Lemak, C.H.; Hearld, L.R.; Wheeler, J.; Sen, B.P.; Menachemi, N. Vertical integration into skilled nursing facilities and hospital readmission rates. J. Healthc. Qual. JHQ 2020, 42, 91-97. [CrossRef]

9. Baechle, C.; Agarwal, A. A framework for the estimation and reduction of hospital readmission penalties using predictive analytics. J. Big Data 2017, 4, 37. [CrossRef]

10. Pugh, J.; Penney, L.S.; Noël, P.H.; Neller, S.; Mader, M.; Finley, E.P.; Lanham, H.J.; Leykum, L. Evidence based processes to prevent readmissions: More is better, a ten-site observational study. BMC Health Serv. Res. 2021, 21, 189. [CrossRef]

11. Kash, B.A.; Baek, J.; Davis, E.; Champagne-Langabeer, T.; Langabeer, J.R. II Review of successful hospital readmission reduction strategies and the role of health information exchange. Int. J. Med. Inform. 2017, 104, 97-104. [CrossRef] [PubMed]

12. McHugh, M.D.; Ma, C. Hospital nursing and 30-day readmissions among Medicare patients with heart failure, acute myocardial infarction, and pneumonia. Med. Care 2013, 51, 52-59. [CrossRef] [PubMed]

13. Lee, E.W. Selecting the best prediction model for readmission. J. Prev. Med. Public Health 2012, 45, 259. [CrossRef] [PubMed]

14. Jasti, H.; Mortensen, E.M.; Obrosky, D.S.; Kapoor, W.N.; Fine, M.J. Causes and risk factors for rehospitalization of patients hospitalized with community-acquired pneumonia. Clin. Infect. Dis. 2008, 46, 550-556. [CrossRef]

15. Donzé, J.; Lipsitz, S.; Bates, D.W.; Schnipper, J.L. Causes and patterns of readmissions in patients with common comorbidities: Retrospective cohort study. BMJ 2013, 347, f7171. [CrossRef]

16. Baker, M.C.; Alberti, P.M.; Tsao, T.-Y.; Fluegge, K.; Howland, R.E.; Haberman, M. Social Determinants Matter For Hospital Readmission Policy: Insights From New York City: Study examines social determinants and hospital readmissions. Health Aff. 2021, 40, 645-654. [CrossRef]

17. Melton, L.D.; Foreman, C.; Scott, E.; McGinnis, M.; Cousins, M. Prioritized post-discharge telephonic outreach reduces hospital readmissions for select high-risk patients. Am. J. Manag. Care 2012, 18, 838-844.

18. Charles, L.; Jensen, L.; Torti, J.M.; Parmar, J.; Dobbs, B.; Tian, P.G.J. Improving transitions from acute care to home among complex older adults using the LACE Index and care coordination. BMJ Open Qual. 2020, 9, e000814. [CrossRef]

19. Garg, A.X.; Adhikari, N.K.; McDonald, H.; Rosas-Arellano, M.P.; Devereaux, P.J.; Beyene, J.; Sam, J.; Haynes, R.B. Effects of computerized clinical decision support systems on practitioner performance and patient outcomes: A systematic review. JAMA 2005, 293, 1223-1238. [CrossRef]

20. Rajkomar, A.; Oren, E.; Chen, K.; Dai, A.M.; Hajaj, N.; Hardt, M.; Liu, P.J.; Liu, X.; Marcus, J.; Sun, M. Scalable and accurate deep learning with electronic health records. NPJ Digit. Med. 2018, 1, 18. [CrossRef]

21. Lai, H.J.; Tan, T.H.; Lin, C.S.; Chen, Y.F.; Lin, H.H. Designing a clinical decision support system to predict readmissions for patients admitted with all-cause conditions. J. Ambient. Intell. Humaniz. Comput. 2020, 1-10. [CrossRef]

22. Wu, F.H.; Lai, H.J.; Lin, H.H.; Chan, P.C.; Tseng, C.M.; Chang, K.M.; Chen, Y.F.; Lin, C.S. Predictive models for detecting patients more likely to develop acute myocardial infarctions. J. Supercomput. 2022, 78, 2043-2071. [CrossRef]

23. Porat, T.; Kostopoulou, O.; Woolley, A.; Delaney, B.C. Eliciting user decision requirements for designing computerized diagnostic support for family physicians. J. Cogn. Eng. Decis. Mak. 2016, 10, 57-73. [CrossRef]

24. Horng, S.; Sontag, D.A.; Halpern, Y.; Jernite, Y.; Shapiro, N.I.; Nathanson, L.A. Creating an automated trigger for sepsis clinical decision support at emergency department triage using machine learning. PLoS ONE 2017, 12, e0174708. [CrossRef]

25. Hsu, J.C.; Chen, Y.F.; Chung, W.S.; Tan, T.H.; Chen, T.S.; Chiang, J.Y. Clinical verification of a clinical decision support system for ventilator weaning. Biomed. Eng. Online 2013, 12, S4. [CrossRef] [PubMed]

26. Luo, G.; Nkoy, F.L.; Gesteland, P.H.; Glasgow, T.S.; Stone, B.L. A systematic review of predictive modeling for bronchiolitis. Int. J. Med. Inform. 2014, 83, 691-714. [CrossRef]

27. Dunn Lopez, K.; Gephart, S.M.; Raszewski, R.; Sousa, V.; Shehorn, L.E.; Abraham, J. Integrative review of clinical decision support for registered nurses in acute care settings. J. Am. Med. Inform. Assoc. 2017, 24, 441-450. [CrossRef]

28. Scheepers-Hoeks, A.-M.J.; Grouls, R.J.; Neef, C.; Ackerman, E.W.; Korsten, E.H. Physicians' responses to clinical decision support on an intensive care unit-Comparison of four different alerting methods. Artif. Intell. Med. 2013, 59, 33-38. [CrossRef]

29. Oluoch, T.; Katana, A.; Kwaro, D.; Santas, X.; Langat, P.; Mwalili, S.; Muthusi, K.; Okeyo, N.; Ojwang, J.K.; Cornet, R. Effect of a clinical decision support system on early action on immunological treatment failure in patients with HIV in Kenya: A cluster randomised controlled trial. Lancet HIV 2016, 3, e76-e84. [CrossRef]

30. Otto, A.K.; Dyer, A.A.; Warren, C.M.; Walkner, M.; Smith, B.M.; Gupta, R.S. The development of a clinical decision support system for the management of pediatric food allergy. Clin. Pediatrics 2017, 56, 571-578. [CrossRef]

31. Ammenwerth, E.; Schnell-Inderst, P.; Machan, C.; Siebert, U. The effect of electronic prescribing on medication errors and adverse drug events: A systematic review. J. Am. Med. Inform. Assoc. 2008, 15, 585-600. [CrossRef] [PubMed]

32. Baypinar, F.; Kingma, H.J.; van der Hoeven, R.T.; Becker, M.L. Physicians' compliance with a clinical decision support system alerting during the prescribing process. J. Med. Syst. 2017, 41, 96. [CrossRef] [PubMed]

33. Arnaud, É.; Elbattah, M.; Gignon, M.; Dequen, G. Deep learning to predict hospitalization at triage: Integration of structured data and unstructured text. In Proceedings of the 2020 IEEE International Conference on Big Data (Big Data), Atlanta, GA, USA, 10-13 December 2020; pp. 4836-4841. 
34. Sánchez-Salmerón, R.; Gómez-Urquiza, J.L.; Albendín-García, L.; Correa-Rodríguez, M.; Martos-Cabrera, M.B.; Velando-Soriano, A.; Suleiman-Martos, N. Machine learning methods applied to triage in emergency services: A systematic review. Int. Emerg. Nurs. 2022, 60, 101109. [CrossRef] [PubMed]

35. Kwon, J.-M.; Jeon, K.-H.; Lee, M.; Kim, K.-H.; Park, J.; Oh, B.-H. Deep learning algorithm to predict need for critical care in pediatric emergency departments. Pediatric Emerg. Care 2021, 37, e988-e994. [CrossRef]

36. Raita, Y.; Goto, T.; Faridi, M.K.; Brown, D.F.; Camargo, C.A.; Hasegawa, K. Emergency department triage prediction of clinical outcomes using machine learning models. Crit. Care 2019, 23, 64. [CrossRef] [PubMed]

37. Hong, W.S.; Haimovich, A.D.; Taylor, R.A. Predicting hospital admission at emergency department triage using machine learning. PLoS ONE 2018, 13, e0201016. [CrossRef] [PubMed]

38. Choi, S.W.; Ko, T.; Hong, K.J.; Kim, K.H. Machine learning-based prediction of Korean triage and acuity scale level in emergency department patients. Healthc. Inform. Res. 2019, 25, 305-312. [CrossRef]

39. Klug, M.; Barash, Y.; Bechler, S.; Resheff, Y.S.; Tron, T.; Ironi, A.; Soffer, S.; Zimlichman, E.; Klang, E. A gradient boosting machine learning model for predicting early mortality in the emergency department triage: Devising a nine-point triage score. J. Gen. Intern. Med. 2020, 35, 220-227. [CrossRef]

40. Navares, R.; Aznarte, J.L. Deep learning architecture to predict daily hospital admissions. Neural Comput. Appl. 2020, 32, 16235-16244. [CrossRef]

41. Chung, A.; Famouri, M.; Hryniowski, A.; Wong, A. COVID-Net Clinical ICU: Enhanced Prediction of ICU Admission for COVID-19 Patients via Explainability and Trust Quantification. arXiv 2021, arXiv:2109.06711.

42. Yu, K.; Yang, Z.; Wu, C.; Huang, Y.; Xie, X. In-hospital resource utilization prediction from electronic medical records with deep learning. Knowl.-Based Syst. 2021, 223, 107052. [CrossRef]

43. Alsinglawi, B.; Alnajjar, F.; Mubin, O.; Novoa, M.; Alorjani, M.; Karajeh, O.; Darwish, O. Predicting Length of Stay for Cardiovascular Hospitalizations in the Intensive Care Unit: Machine Learning Approach. In Proceedings of the 2020 42nd Annual International Conference of the IEEE Engineering in Medicine \& Biology Society (EMBC), Montreal, QC, Canada, 20-24 July 2020; pp. 5442-5445.

44. Xie, J.; Zhang, B.; Ma, J.; Zeng, D.; Lo-Ciganic, J. Readmission prediction for patients with heterogeneous medical history: A trajectory-based deep learning approach. ACM Trans. Manag. Inf. Syst. TMIS 2021, 13, 1-27. [CrossRef]

45. Choudhury, A.; Greene, C.M. Evaluating patient readmission risk: A predictive analytics approach. arXiv 2018, arXiv:1812.11028. [CrossRef]

46. Jiang, S.; Chin, K.S.; Qu, G.; Tsui, K.L. An integrated machine learning framework for hospital readmission prediction. Knowl.Based Syst. 2018, 146, 73-90. [CrossRef]

47. Du, G.; Zhang, J.; Luo, Z.; Ma, F.; Ma, L.; Li, S. Joint imbalanced classification and feature selection for hospital readmissions Knowl.-Based Syst. 2020, 200, 106020. [CrossRef]

48. Du, G.; Zhang, J.; Ma, F.; Zhao, M.; Lin, Y.; Li, S. Towards graph-based class-imbalance learning for hospital readmission. Expert Syst. Appl. 2021, 176, 114791. [CrossRef]

49. Junqueira, A.R.B.; Mirza, F.; Baig, M.M. A machine learning model for predicting ICU readmissions and key risk factors: Analysis from a longitudinal health records. Health Technol. 2019, 9, 297-309. [CrossRef]

50. Ryu, B.; Yoo, S.; Kim, S.; Choi, J. Development of Prediction Models for Unplanned Hospital Readmission within 30 Days Based on Common Data Model: A Feasibility Study. Methods Inf. Med. 2021, 60, e65-e75.

51. Weinreich, M.; Nguyen, O.K.; Wang, D.; Mayo, H.; Mortensen, E.M.; Halm, E.A.; Makam, A.N. Predicting the risk of readmission in pneumonia. A systematic review of model performance. Ann. Am. Thorac. Soc. 2016, 13, 1607-1614. [CrossRef]

52. Capelastegui, A.; Yandiola, P.P.E.; Quintana, J.M.; Bilbao, A.; Diez, R.; Pascual, S.; Pulido, E.; Egurrola, M. Predictors of short-term rehospitalization following discharge of patients hospitalized with community-acquired pneumonia. Chest 2009, 136, 1079-1085. [CrossRef]

53. Mather, J.F.; Fortunato, G.J.; Ash, J.L.; Davis, M.J.; Kumar, A. Prediction of pneumonia 30-day readmissions: A single-center attempt to increase model performance. Respir. Care 2014, 59, 199-208. [CrossRef] [PubMed]

54. Makam, A.N.; Nguyen, O.K.; Clark, C.; Zhang, S.; Xie, B.; Weinreich, M.; Mortensen, E.M.; Halm, E.A. Predicting 30-day pneumonia readmissions using electronic health record data. J. Hosp. Med. 2017, 12, 209. [CrossRef] [PubMed]

55. Hatipoğlu, U.; Wells, B.J.; Chagin, K.; Joshi, D.; Milinovich, A.; Rothberg, M.B. Predicting 30-day all-cause readmission risk for subjects admitted with pneumonia at the point of care. Respir. Care 2018, 63, 43-49. [CrossRef]

56. Lai, H.J.; Chan, P.C.; Lin, H.H.; Chen, Y.F.; Lin, C.S.; Hsu, J.C. A web-based decision support system for predicting readmission of pneumonia patients after discharge. In Proceedings of the 2018 IEEE International Conference on Systems, Man, and Cybernetics (SMC), Miyazaki, Japan, 7-10 October 2018; pp. 2305-2310.

57. Chang, C.C.; Liao, C.C.; Chen, T.L. Perioperative medicine and Taiwan National Health Insurance Research Database. Acta Anaesthesiol. Taiwanica 2016, 54, 93-96. [CrossRef]

58. Farquad, M.; Bose, I. Preprocessing unbalanced data using support vector machine. Decis. Support Syst. 2012, 53, 226-233. [CrossRef]

59. Hu, J.; Yang, H.; Lyu, M.R.; King, I.; So, A.M.C. Online nonlinear AUC maximization for imbalanced data sets. IEEE Trans. Neural Netw. Learn. Syst. 2017, 29, 882-895. [CrossRef] [PubMed] 
60. Chen, Y.F.; Lin, C.S.; Wang, K.A.; Rahman, L.O.A.; Lee, D.J.; Chung, W.S.; Lin, H.H. Design of a clinical decision support system for fracture prediction using imbalanced dataset. J. Healthc. Eng. 2018, 2018, 9621640. [CrossRef]

61. Charlson, M.E.; Pompei, P.; Ales, K.L.; MacKenzie, C.R. A new method of classifying prognostic comorbidity in longitudinal studies: Development and validation. J. Chronic Dis. 1987, 40, 373-383. [CrossRef]

62. Lin, W.C.; Tsai, C.F.; Hu, Y.H.; Jhang, J.S. Clustering-based undersampling in class-imbalanced data. Inf. Sci. 2017, 409, 17-26. [CrossRef]

63. Chang, C.C.; Lin, C.J. LIBSVM: A Library for Support Vector Machines. Available online: https://www.csie.ntu.edu.tw/ \{\}cjlin/ libsvm/faq.html\#f203 (accessed on 29 December 2021).

64. Chen, Y.F.; Huang, P.C.; Lin, K.C.; Lin, H.H.; Wang, L.E.; Cheng, C.C.; Chen, T.P.; Chan, Y.K.; Chiang, J.Y. Semi-automatic segmentation and classification of pap smear cells. IEEE J. Biomed. Health Inform. 2013, 18, 94-108. [CrossRef]

65. Pedregosa, F.; Varoquaux, G.; Gramfort, A.; Michel, V.; Thirion, B.; Grisel, O.; Blondel, M.; Prettenhofer, P.; Weiss, R.; Dubourg, V. Scikit-learn: Machine learning in Python. J. Mach. Learn. Res. 2011, 12, 2825-2830.

66. Abadi, M.; Agarwal, A.; Barham, P.; Brevdo, E.; Chen, Z.; Citro, C.; Corrado, G.S.; Davis, A.; Dean, J.; Devin, M. TensorFlow: Large-scale Machine Learning on Heterogeneous Systems. arXiv Prepr. 2016, arXiv:1603.04467.

67. Pilgrim, M.; Willison, S. Dive into Python 3; Springer: Berlin/Heidelberg, Germany, 2009; Volume 2.

68. Chen, Y.F.; Lin, C.S.; Hong, C.F.; Lee, D.J.; Sun, C.M.; Lin, H.H. Design of a clinical decision support system for predicting erectile dysfunction in men using NHIRD dataset. IEEE J. Biomed. Health Inform. 2019, 23, 2127-2137. [CrossRef] [PubMed]

69. Petersen, P.T.; Egelund, G.B.; Jensen, A.V.; Andersen, S.B.; Pedersen, M.F.; Rohde, G.; Ravn, P. Associations between biomarkers at discharge and co-morbidities and risk of readmission after community-acquired pneumonia: A retrospective cohort study. Eur. J. Clin. Microbiol. Infect. Dis. 2018, 37, 1103-1111. [CrossRef] [PubMed]

70. Dong, T.; Cursio, J.F.; Qadir, S.; Lindenauer, P.K.; Ruhnke, G.W. Discharge disposition as an independent predictor of readmission among patients hospitalised for community-acquired pneumonia. Int. J. Clin. Pract. 2017, 71, e12935. [CrossRef] [PubMed]

71. Danso, S.O.; Zeng, Z.; Muniz-Terrera, G.; Ritchie, C.W. Developing an explainable machine learning-based personalised dementia risk prediction model: A transfer learning approach with ensemble learning algorithms. Front. Big Data 2021, 4, 21. [CrossRef] [PubMed]

72. Zhuang, F.; Qi, Z.; Duan, K.; Xi, D.; Zhu, Y.; Zhu, H.; Xiong, H.; He, Q. A comprehensive survey on transfer learning. Proc. IEEE 2020, 109, 43-76. [CrossRef]

73. Feuz, K.D.; Cook, D.J. Collegial activity learning between heterogeneous sensors. Knowl. Inf. Syst. 2017, 53, 337-364. [CrossRef] 